\title{
A Time Complexity Lower Bound for Adaptive Mutual Exclusion*
}

\author{
Yong-Jik Kim \\ Google Inc. \\ 1600 Amphitheatre Parkway \\ Mountain View, CA 94043 \\ Email: yongjik@gmail.com
}

\author{
James H. Anderson \\ Department of Computer Science \\ University of North Carolina at Chapel Hill \\ Chapel Hill, NC 27599-3175 \\ Email: anderson@cs.unc.edu
}

September 2005, revised May 2007, December 2010, and August 2011

\begin{abstract}
We consider the time complexity of adaptive mutual exclusion algorithms, where "time" is measured by counting the number of remote memory references required per critical-section access. For systems that support (only) read, write, and comparison primitives (such as compare-and-swap), we establish a lower bound that precludes a deterministic algorithm with $o(k)$ time complexity, where $k$ is point contention. In particular, it is impossible to construct a deterministic $O(\log k)$ algorithm based on such primitives.
\end{abstract}

\footnotetext{
${ }^{*}$ Work supported by NSF grants CCR 9732916, CCR 9972211, CCR 9988327, ITR 0082866, and CCR 0208289. This work was presented in preliminary form at the 15th International Symposium on Distributed Computing [30], where it received the best student paper award.
} 


\section{Introduction}

In this paper, we consider the time complexity of adaptive mutual exclusion algorithms. A mutual exclusion algorithm is adaptive if its time complexity is a function of the number of contending processes [7, 15, 25, 28, 31, 34, 35]. Two widely used definitions of contention are "interval contention" and "point contention" [1]. ${ }^{1}$ The interval contention over a computation $H$ is the number of processes that are active in $H$, i.e., that execute outside of their noncritical sections. The point contention over $H$ is the maximum number of processes that are active at the same state in $H$. Throughout this paper, we let $N$ denote the number of processes in the system. Also, unless stated otherwise, $k$ denotes the point contention experienced by an arbitrary process while it is active. (Note that point contention is always at most interval contention. Hence, our lower bound result, proved in terms of point contention, automatically applies to interval contention as well.)

The time complexity measure considered in this paper is motivated by work on local-spin synchronization algorithms. In local-spin algorithms, all busy waiting is by means of read-only loops in which one or more locallyaccessible "spin variables" are repeatedly tested. The ability to locally access a shared variable is provided on both distributed shared-memory (DSM) and cache-coherent (CC) machines, as illustrated in Figure 1. In a DSM machine, each processor has its own memory module that can be accessed without accessing the global interconnection network. On such a machine, a shared variable can be made locally accessible by storing it in a local memory module. In a CC machine, each processor has a private cache, and some hardware protocol is used to enforce cache consistency (i.e., to ensure that all copies of the same variable in different local caches are consistent). On such a machine, a shared variable becomes locally accessible by migrating to a local cache line. $^{2}$

Because our main interest is local-spin algorithms, we determine the time complexity of a mutual exclusion algorithm by counting the number of remote memory references generated by one process to enter and then exit its critical section. A remote memory reference (RMR) is a memory access that requires a traversal of the global processors-to-memory interconnect. This complexity measure is known as the RMR time complexity measure [5].

In prior work, we presented an adaptive mutual exclusion algorithm with $O(\min (k, \log N))$ RMR time complexity that is based only on reads and writes [31]. (A similar algorithm has also been presented by Afek et al. [3].) Our algorithm is based on Yang and Anderson's (non-adaptive) algorithm [39], which has $\Theta(\log N)$ RMR time complexity. In other prior work, we established a worst-case RMR time bound of $\Omega(\log N / \log \log N)$ for mutual exclusion algorithms (adaptive or not) based on reads, writes, or comparison primitives ${ }^{3}$ such as $^{2}$ test-and-set and compare-and-swap [4]. We also conjectured that this bound can be improved to $\Omega(\log N)$, which would then prove the optimality of Yang and Anderson's algorithm. Recently, Attiya et al. answered this conjecture in the affirmative by proving $\Omega(\log N)$ RMR time bound for a wide class of algorithms including mutual exclusion [9], with one exception: their result does not apply to CC machines that use a write-update protocol. ${ }^{4}$ These two results show that the $\Theta(\log N)$ worst-case RMR time complexity of our $O(\min (k, \log N))$

\footnotetext{
${ }^{1}$ Another notion of contention, namely "step contention" [8], is used primarily in the study of obstruction-free algorithms [26]. We do not concern ourselves with step contention in this paper.

${ }^{2}$ Most modern multiprocessor systems are CC machines. However, DSM architectures are still being used in the embeddedsystems domain, where simpler computing technology often must be used due to cost/power limitations, and the non-deterministic nature of cache hardware is sometimes undesirable. For example, the Cradle Technologies CT3600 multicore digital signal processor has on-chip memory, but no data cache. If several of these processors are interconnected, then the resulting platform is a DSM platform as considered here (with the exception that cores on the same chip can access the same local memory). A well-known older example of a DSM machine is the BBN Butterfly 1, which was considered by Mellor-Crummey and Scott [33] in their performance studies involving local-spin algorithms.

${ }^{3} \mathrm{~A}$ comparison primitive conditionally updates a shared variable after first testing that its value equals a prescribed value.

${ }^{4}$ In a write-update CC machine, when a processor updates a variable shared among multiple processors' caches, the updated
} 


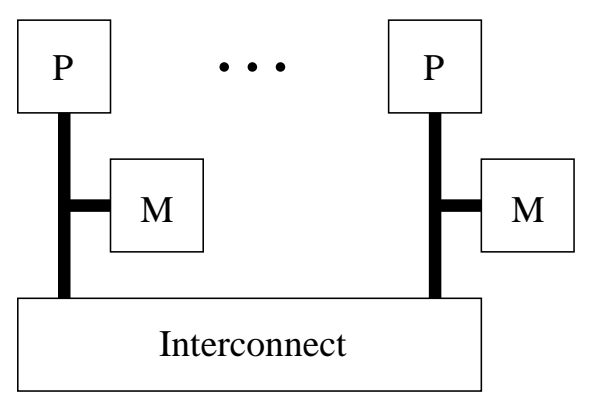

(a)

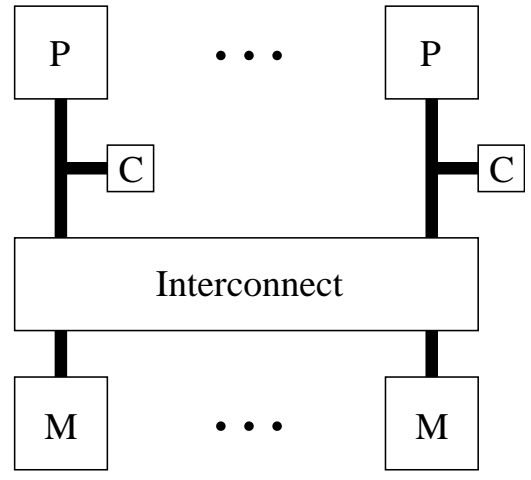

(b)

Figure 1: (a) DSM model. (b) CC model. In both insets, 'P' denotes a processor, 'C' a cache, and ' $\mathrm{M}$ ' a memory module.

algorithm [31] is optimal for DSM machines and write-invalidate CC machines, and close to optimal (specifically, within a factor of $\Theta(\log \log N))$ for write-update $\mathrm{CC}$ machines.

These two lower bounds do not mention $k$, so they tell us very little about RMR time complexity under low contention. The only bounds involving $k$ that directly follow are obtained by substituting $k$ for $n$ in their proofs. In particular, our $\Omega(\log N / \log \log N)$ lower bound is established by inductively considering longer and longer computations, the first of which involves $N$ processes, and the last of which may involve fewer processes. If we start instead with $k$ process, then a computation is obtained with $O(k)$ processes (and hence $O(k)$ point contention at each state) in which some process performs $\Omega(\log k / \log \log k)$ remote references. A similar argument, applied to the proof of the $\Omega(\log N)$ bound [9], yields an $\Omega(\log k)$ time bound.

This suggests two interesting possibilities: in all likelihood, either $\Omega(\min (k, \log N))$ is in fact a tight lower bound (i.e., the algorithm in [31] is optimal), or it is possible to design an adaptive algorithm with $O(\log k)$ RMR time complexity (i.e., $\Omega(\log k)$ is tight). Indeed, the problem of designing an $O(\log k)$ algorithm using only reads and writes has been mentioned in at least two papers $[7,31]$.

In this paper, we show that an $O(\log k)$ algorithm in fact does not exist. In particular, we prove the following.

Given any $k$, define $\bar{N}=\bar{N}(k)=(2 k+4)^{2\left(2^{k}-1\right)}$. For any $N \geq \bar{N}$, and for any $N$-process mutual exclusion algorithm based on reads, writes, or comparison primitives, a computation exists involving $\Theta(k)$ processes in which some process performs $\Omega(k)$ remote memory references to enter and exit its critical section.

There exists one exception to our result: our lower bound does not apply to write-update CC machines that have the ability to execute failed comparison events on cached variables without generating interconnect traffic. (See Section 2.3 for details.) In fact, an algorithm with $O(1)$ time complexity in such systems is presented in [4].

Our proof of this result extends techniques used by us and others in several earlier papers $[2,4,6,13,14$, $16,36]$.

value propagates to these caches. On the other hand, a write-invalidate machine simply invalidates remote copies in such a case; a subsequent read by another processor thus generates interconnect traffic to access the updated value. 
Related work. Since the publication of these results in preliminary form [30], a number of papers have been published that address issues pertaining to RMR time complexity $[9,10,11,12,17,18,19,21,22,23,24,25$, 27, 28, 32, 37]. Of these, Attiya et al. [9] and Hendler and Woelfel [25] are of direct relevance to the focus of this paper. As mentioned above, Attiya et al. [9] proved a tight RMR lower bound for mutual exclusion for most class of machines. Their result extends ideas used by Fan and Lynch [20], which proved a lower bound of $\Omega(\log N)$ operations (per process) under a different model, namely, the state change cost model. It is worth noting that these two papers use information-theoretic arguments, which allow processes to learn about other running processes, unlike our result presented in this paper, where limiting the "information flow" is crucial.

Recently, Hendler and Woelfel devised a randomized mutual exclusion algorithm with expected $O(\log N /$ $\log \log N)$ RMR time complexity [24], and a randomized adaptive algorithm with an expected amortized $O(\log k /$ $\log \log k$ ) RMR time complexity [25]. Thus, randomized algorithms can perform better than deterministic ones.

RMR time complexity has been also studied in light of other related algorithms such as leader election [23], reader-writer locks [11, 12], group mutual exclusion [10, 19, 29], $k$-exclusion [17], first-come first-served (FCFS) mutual exclusion [18, 27, 37], abortable mutual exclusion [28], and implementing comparison primitives using reads and writes [22].

The rest of the paper is organized as follows. In Section 2, our system model is defined. Our lower bound proof is then sketched in Section 3. A formal proof of it is given in Section 4. We conclude in Section 5.

\section{Definitions}

In this section, we provide definitions pertaining to atomic shared-memory systems that will be used in obtaining our lower bound. In the following subsections, we define our model of an atomic shared-memory system (Section 2.1), state the properties required of a mutual exclusion algorithm implemented within this model (Section 2.2), and present a categorization of events that allows us to accurately deduce the network traffic generated by an algorithm in a system with coherent caches (Section 2.3). The same model was used earlier by us to establish the previously-mentioned $\Omega(\log N / \log \log N)$ lower bound [4]. Therefore, most of the material in this section is taken directly from [4].

\subsection{Atomic Shared-Memory Systems}

Our model of an atomic shared-memory system is similar to that used by Anderson and Yang [6].

An atomic shared-memory system $\mathcal{S}=(C, P, V)$ consists of a set of computations $C$, a set of processes $P$, and a set of variables $V$. A computation is a finite sequence of events. To complete the definition of an atomic shared-memory system, we must formally define the notion of an "event" and state the requirements to which events and computations are subject. This is done in the remainder of this subsection.

Each variable is local to at most one process and is remote to all other processes. (Note that we allow variables that are remote to all processes; thus, our model applies to both DSM and CC systems.) The locality relationship is static, i.e., it does not change during a computation. A local variable may be shared; that is, a process may access local variables of other processes. An initial value is associated with each variable.

We assume that each process $p \in P$ asynchronously executes a series of atomic statements, as follows.

Step 1. Determine an atomic statement to execute, based on $p$ 's internal state (i.e., its private variables and program counter). This step may not access any shared variable. 
Step 2. Execute the selected statement atomically. The code may contain at most one access to a remote variable, and an unlimited number of accesses to any of $p$ 's local variables (private or shared).

Step 3. Check for program termination (which may be indicated by a private variable, for example). If the program has terminated, then stop execution. Otherwise, go back to Step 1.

Events, informally considered. Below, formal definitions pertaining to events are given; here, we present an informal discussion to motivate these definitions. Informally, an event is a particular execution of an atomic statement (Step 2 above) of some process that involves reading and/or writing one or more variables. An event is local if it does not access any remote variables, and is remote otherwise. An event is executed by a particular process, and may access at most one variable that is remote to that process (by reading, writing, or executing a comparison primitive), plus any number of local (shared or private) variables. Thus, we allow arbitrarily powerful operations on local variables. Since our proof applies to systems with reads, writes, and comparison primitives, it is important to formally define the notion of a comparison primitive. We define a comparison primitive to be an atomic operation on a shared variable $v$ expressible using the following pseudo-code.

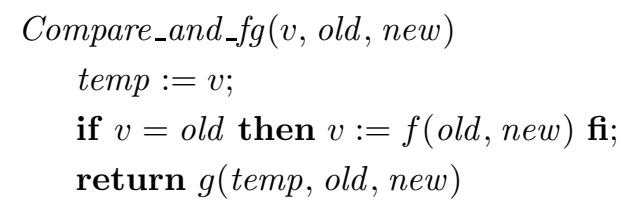

For example, compare-and-swap can be defined by defining $f($ old, new $)=$ new and $g(t e m p$, old, new $)=$ old. We call an execution of such a primitive a comparison event. As we shall see, our formal definition of a comparison event, which is given later in this section, generalizes the functionality encompassed by the pseudocode above by allowing arbitrarily many local shared variables to be accessed.

As an example, assume that variables $a, b$, and $c$ are local to process $p$ and variables $x$ and $y$ are remote to $p$. Then, the following atomic statements by $p$ are allowed in our model.

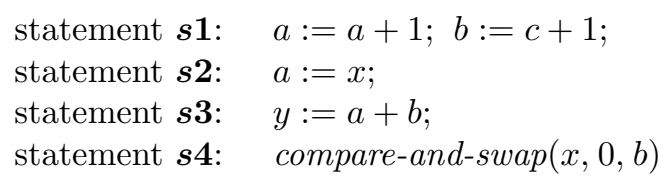

For example, if every variable has an initial value of 0 , and if these four statements are executed in order, then we will have the following four events.

$e 1: p$ reads 0 from $a$, writes 1 to $a$, reads 0 from $c$, and writes 1 to $b$;

/* local event $* /$

$e 2: p$ reads 0 from $x$ and writes 0 to $a$;

$e 3$ : $p$ reads 0 from $a$, reads 1 from $b$, and writes 1 to $y$;

$/ *$ remote read from $x * /$

/* remote write to $y * /$

$e 4$ : $p$ reads 0 from $x$, reads 1 from $b$, and writes 1 to $x \quad / *$ comparison primitive execution on $x * /$

On the other hand, the following atomic statements by $p$ are not allowed in our model, because $s 5$ accesses two remote variables at once, and $s 6$ and $s 7$ cannot be expressed as a comparison primitive.

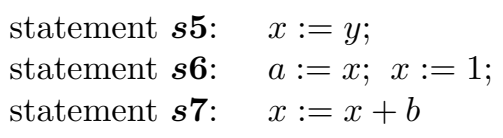

/* accesses two remote variables $*$ / /*fetch-and-store (swap) on a remote variable */ /* fetch-and-add on a remote variable $*$ / 
Describing each event as in the preceding examples is inconvenient, ambiguous, and prone to error. For example, if statement $s 7$ is executed when $x=0 \wedge b=1$ holds, then the resulting event can be described in the same way as $e 4$ is. (Thus, $e 4$ is allowed as an execution of $s 4$, yet disallowed as an execution of $s 7$.) In order to systematically represent the class of allowed events, we need a more refined formalism.

Definitions pertaining to atomic statements. We classify each atomic statement based on its operation, which determines which remote variable is accessed, and what primitive is used to access it. An atomic statement must execute one of the following operations: $\perp$ (which represents a local event), $\operatorname{read}(v)$, write $(v)$, or compare $(v, \alpha)$, where $v$ is a variable in $V$ and $\alpha$ is a value from the value domain of $v$. Moreover, the exact type of the operation (including the values $v$ and $\alpha$, where applicable) must be determined by Step 1, before any shared variable is accessed (by Step 2). This property is formally stated in Property P3, given later.

For example, if $a$ is a shared variable, local to process $p$, and $x$ and $y$ are remote variables, then the following statement is not a valid atomic statement for $p$.

$$
\text { statement } s 8: \quad \text { if } a=0 \text { then } x:=1 \text { else } y:=1 \mathrm{fi}
$$

This is because $s 8$ may execute either write $(x)$ or write $(y)$ as its operation, depending on the value possibly written to $a$ by other processes. That is, its operation cannot be determined at Step 1 . On the other hand, the following statement is allowed, because its operation is fixed as write $(x)$.

$$
\text { statement } s 9: \quad \text { if } a=0 \text { then } x:=1 \text { else } x:=2 \mathrm{fi}
$$

As yet another example, if $k$ is a private variable, then the atomicity in the following code segment is acceptable: in it, there are two distinct atomic statements, one of which will be executed depending on $k$ 's value. (Compare with $s 8$.)

$$
\begin{array}{ccc} 
& \text { if } k=0 \text { then } \quad / * \text { processed at Step } 1 * / \\
\text { statement } \boldsymbol{s} \mathbf{1 0}: & x:=1 \\
& \text { else } \\
\text { statement } \boldsymbol{s} \mathbf{1 1}: & \quad y:=1 \\
& \text { fi }
\end{array}
$$

Similarly, if $k$ is a private variable and $a$ and $b$ are shared local variables, then the following is a valid atomic statement with operation compare $(x, \alpha)$, where $\alpha$ is the value of $k$ just before the execution of $s 12$. However, if $k$ is a shared variable, then $s 12$ is disallowed, because its operation cannot be determined by examining private variables only.

statement $s$ 12: if $x=k$ then $x:=a+b+1 ; k:=0 \mathrm{fi}$

Definitions pertaining to events. We now formally define an event and state its requirements. An event is a particular execution of an atomic statement (by a particular process). For brevity, we sometimes use $e_{p}$ to denote an event executed by process $p$. The operation of $e$, denoted $o p(e)$, is the operation of the corresponding atomic statement, as defined above. We use Rvar(e) (respectively, Wvar $(e)$ ) to denote the set of variables read (respectively, written) by $e$. Rvar $(e)$ and $W v a r(e)$ need not be disjoint, and may contain an arbitrary number of local variables. We also define $\operatorname{var}(e)$, the set of all variables accessed by $e$, to be $\operatorname{Rvar}(e) \cup W v a r(e)$. We also say that a computation $H$ contains a write (respectively, read) of $v$ if $H$ contains some event that writes (respectively, reads) $v$. 
The values read from variables in $R v \operatorname{var}(e)$ or written into variables in $W v a r(e)$ are a part of $e$ 's specification. (For example, if process $p$ executes an identical atomic statement that reads variable $v$ in two different computations, but reads a different value in either case, then these two are considered different events.) Clearly, in a valid computation, an event $e$ must read from each $v \in R v a r(e)$ the value that $v$ held just before the execution of $e$. This requirement is formally stated in Property $\mathrm{P} 4$ below.

Our lower bound is dependent on the Atomicity property stated below. This assumption requires each remote event to be an atomic read operation, an atomic write operation, or a comparison-primitive execution.

Atomicity property: The operation of each event $e$ by a process $p$ must satisfy one of the conditions below.

- If $o p(e)=\perp$, then $e$ does not access any remote variables. (That is, all variables in $\operatorname{var}(e)$ are local to $p$.) In this case, we call $e$ a local event.

- If $o p(e)=\operatorname{read}(v)$, then $e$ reads exactly one remote variable, which must be $v$, and does not write any remote variable. (That is, $v \in R \operatorname{var}(e), v \notin \operatorname{Wvar}(e)$, and all other variables [if any] in $\operatorname{var}(e)$ are local to p.) In this case, $e$ is called a remote read event.

- If $o p(e)=$ write $(v)$, then $e$ writes exactly one remote variable, which must be $v$, and does not read any remote variable. (That is, $v \in \operatorname{Wvar}(e), v \notin R \operatorname{var}(e)$, and all other variables [if any] in $\operatorname{var}(e)$ are local to p.) In this case, $e$ is called a remote write event.

- If $o p(e)=\operatorname{compare}(v, \alpha)$, then $e$ reads exactly one remote variable, which must be $v$. We say that $e$ is a comparison event in this case. Comparison events must be either successful or unsuccessful.

- $e$ is a successful comparison event if $e$ reads the value $\alpha$ from $v$ and writes some different value $\beta$ $(\neq \alpha)$ to $v$.

- $e$ is an unsuccessful comparison event if $e$ does not write $v$, i.e., $v \notin W v a r(e)$ holds.

In either case, $e$ does not write or read any other remote variable.

Our notion of an unsuccessful comparison event includes both comparison-primitive invocations that fail (i.e., $v \neq$ old in the pseudo-code given for Compare_and_fg above) and also those that do not fail but leave the remote variable that is accessed unchanged (i.e., $v=$ old $\wedge v=f($ old, new)). In the latter case, we simply assume that the remote variable $v$ is not written. We categorize both cases as unsuccessful comparison events because this allows us to simplify certain cases in our lower bound proof. (On the other hand, we do allow a remote write event on $v$ to preserve the value of $v$, i.e., to write the same value as $v$ had before the event.)

Note that the Atomicity property allows arbitrarily powerful operations on local (shared) variables. For example, if variable $v$, ranging over $\{0, \ldots, 10\}$, is remote to process $p$, and arrays $a[1 . .10]$ and $b[1 . .10]$ are local to $p$, then an execution of the following statement is a valid event $e$ by $p$ with operation compare $(v, 0)$.

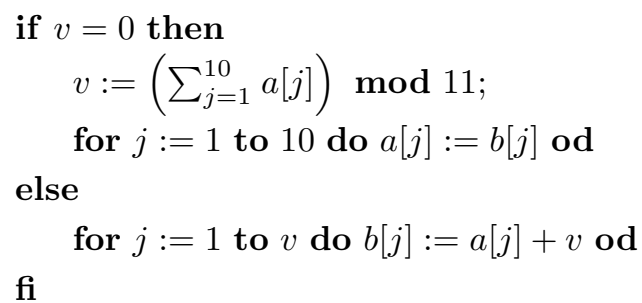


In this case, $\operatorname{Wvar}(e)$ is $\{v, a[1 . .10]\}$ if $e$ reads $v=0$ and writes a nonzero value (i.e., $e$ is a successful comparison event), $\{a[1 . .10]\}$ if $e$ reads and writes $v=0,{ }^{5}$ and $\{b[1 . . v]\}$ if $e$ reads a value between 1 and 10 from $v$.

It is important to note that, saying that an event $e_{p}$ writes (reads) a variable $v$ is not equivalent to saying that $e_{p}$ is a remote write (read) event on $v ; e_{p}$ may also write (read) $v$ if $v$ is local to process $p$, or if $p$ is a comparison event that accesses $v$.

We say that two events $e$ and $f$ are congruent, denoted $e \sim f$, if and only if the following conditions are met.

- $e$ and $f$ are executed by the same process;

- $o p(e)=o p(f)$, where equality means that both operations are the same with the same arguments ( $v$ and/or $\alpha)$.

Informally, two events are congruent if they execute the same operation on the same remote variable. For read and write events, the values read or written may be different. For comparison events, the values read or written (if successful) may be different, but the parameter $\alpha$ must be the same. (It is possible that a successful comparison event is congruent to an unsuccessful one.) Note that $e$ and $f$ may access different local variables.

Definitions pertaining to computations. The definitions given until now have mostly focused on events. We now present requirements and definitions pertaining to computations.

The value of variable $v$ at the end of computation $H$, denoted value $(v, H)$, is the last value written to $v$ in $H$ (or the initial value of $v$ if $v$ is not written in $H$ ). The last event to write to $v$ in $H$ is denoted writer $\operatorname{event}(v, H),{ }^{6}$ and the process that executes it is denoted writer $(v, H)$. If $v$ is not written by any event in $H$, then we let $\operatorname{writer}(v, H)=\perp$ and writer_event $(v, H)=\perp$.

We use $\langle e, \ldots\rangle$ to denote a computation that begins with the event $e,\langle e, \ldots, f\rangle$ to denote a computation beginning with event $e$ and ending with event $f$, and \langle\rangle to denote the empty computation. We use $H \circ G$ to denote the computation obtained by concatenating computations $H$ and $G$. An extension of computation $H$ is a computation of which $H$ is a prefix. For a computation $H$ and a set of processes $Y, H \mid Y$ denotes the subcomputation of $H$ that contains all events in $H$ of processes in $Y{ }^{7}$ A computation $H$ is a $Y$-computation if and only if $H=H \mid Y$. For simplicity, we abbreviate the preceding definitions when applied to a singleton set of processes. For example, if $Y=\{p\}$, then we use $H \mid p$ to mean $H \mid\{p\}$ and $p$-computation to mean $\{p\}$-computation. Two computations $H$ and $G$ are congruent, denoted $H \sim G$, if either both $H$ and $G$ are empty, or if $H=\langle e\rangle \circ H^{\prime}$ and $G=\langle f\rangle \circ G^{\prime}$, where $e \sim f$ and $H^{\prime} \sim G^{\prime}$.

Until this point, we have placed no restrictions on the set of computations $C$ of an atomic shared-memory system $\mathcal{S}=(C, P, V)$ (other than restrictions pertaining to the kinds of events that are allowed in an individual computation). The restrictions we require are as follows.

P1: If $H \in C$ and $G$ is a prefix of $H$, then $G \in C$.

- Informally, every prefix of a valid computation is also a valid computation.

P2: If $H \circ\left\langle e_{p}\right\rangle \in C, G \in C, G|p=H| p$, and if value $(v, G)=\operatorname{value}(v, H)$ holds for all $v \in \operatorname{Rvar}\left(e_{p}\right)$, then $G \circ\left\langle e_{p}\right\rangle \in C$.

\footnotetext{
${ }^{5}$ In other words, we consider $v$ as not written by $e$.

${ }^{6}$ Although our definition of an event allows multiple instances of the same event, we assume that such instances are distinguishable from each other. (For simplicity, we do not extend our notion of an event to include an additional identifier for distinguishability.)

${ }^{7}$ The subcomputation $H \mid Y$ is not necessarily a valid computation. However, we can always consider $H \mid Y$ to be a computation in a technical sense, i.e., it is a sequence of events.
} 
- Informally, if two computations $H$ and $G$ are not distinguishable to process $p$, if $p$ can execute event $e_{p}$ after $H$, and if all variables in $\operatorname{Rvar}\left(e_{p}\right)$ have the same values after $H$ and $G$, then $p$ can execute $e_{p}$ after $G$.

P3: If $H \circ\left\langle e_{p}\right\rangle \in C, G \in C$, and $G|p=H| p$, then $G \circ\left\langle e_{p}^{\prime}\right\rangle \in C$ for some event $e_{p}^{\prime}$ such that $e_{p} \sim e_{p}^{\prime}$. - Informally, if two computations $H$ and $G$ are not distinguishable to process $p$, and if $p$ can execute event $e_{p}$ after $H$, then $p$ can execute a congruent event after $G$.

P4: For any $H \in C, H \circ\left\langle e_{p}\right\rangle \in C$ implies that $e$ reads the value of value $(v, H)$ from $v$, for all $v \in R v a r\left(e_{p}\right)$. - Informally, only the last value written to a variable can be read.

P5: For any $H \in C$, if both $H \circ\left\langle e_{p}\right\rangle \in C$ and $H \circ\left\langle e_{p}^{\prime}\right\rangle \in C$ hold for two events $e_{p}$ and $e_{p}^{\prime}$, then $e_{p}=e_{p}^{\prime}$. - Informally, each process is deterministic. This property is included in order to simplify bookkeeping in our proofs.

Note that Property P3 precisely defines the class of allowed events. (For example, statement $s 8$ is disallowed because it violates P3.) P3 and P5 imply that, if $p$ cannot distinguish between two computations, then any atomic statement $p$ is about to execute immediately after them must result in congruent events. Hence, congruence of execution is a necessary condition for every allowed atomic statement. We invite the reader to verify that this is indeed satisfied by statements $s 1-s 4$ and $s 9-s 12$.

\subsection{Mutual Exclusion Systems}

We now define a special kind of atomic shared-memory system, namely (atomic) mutual exclusion systems, which are our main interest. An atomic mutual exclusion system $\mathcal{S}=(C, P, V)$ is an atomic shared-memory system that satisfies the properties below.

Each process $p$ has a local auxiliary variable $s_{a t}$ that represents which section in the mutual exclusion

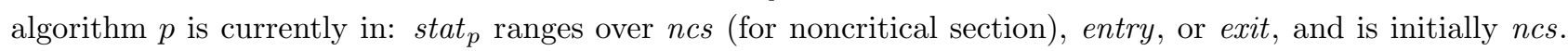
(For simplicity, we assume that each critical-section execution is vacuous.) Process $p$ also has three "dummy" auxiliary variables $n c s_{p}$, entry $y_{p}$, and exit $_{p}$. These variables are accessed only by the following atomic statements, which only $p$ may execute. (For simplicity, we use Enter ${ }_{p}, C S_{p}$, and Exit $t_{p}$ to denote both the statements given below and the events representing their execution.)

$$
\begin{array}{lll}
\text { Enter }_{\boldsymbol{p}}: & \text { stat }_{p}:=\text { entry } ; & \text { entry }_{p}:=0 \\
\text { CS }_{\boldsymbol{p}}: & \text { stat }_{p}:=\text { exit } ; & \text { exit }_{p}:=0 \\
\text { Exit }_{\boldsymbol{p}}: & \text { stat }_{p}:=n c s ; & \text { ncs }_{p}:=0
\end{array}
$$

Enter $_{p}$ causes $p$ to transit from its noncritical section to its entry section. $C S_{p}$ causes $p$ to transit from its entry section to its exit section. ${ }^{8}$ Exit $_{p}$ causes $p$ to transit from its exit section to its noncritical section. This behavior is depicted in Figure 2.

We define variables entry , exit $_{p}$, and $n c s_{p}$ to be remote to all processes. This assumption allows us to simplify bookkeeping, because it implies that each of Enter ${ }_{p}, C S_{p}$, and Exit $t_{p}$ is congruent only to itself. (This is the sole purpose of defining these three variables.)

The allowable transitions of stat $_{p}$ are as follows: for all $H \in C$,

$$
\begin{aligned}
& H \circ\left\langle\text { Enter }_{p}\right\rangle \in C \text { if and only if } \operatorname{value}\left(\text { stat }_{p}, H\right)=n c s \text {; } \\
& H \circ\left\langle C S_{p}\right\rangle \in C \quad \text { only if } \quad \operatorname{value}\left(\operatorname{stat}_{p}, H\right)=\text { entry; } \\
& H \circ\left\langle\text { Exit }_{p}\right\rangle \in C \quad \text { only if } \quad \text { value }\left(\operatorname{stat}_{p}, H\right)=\text { exit. }
\end{aligned}
$$

\footnotetext{
${ }^{8}$ Each critical-section execution of $p$ is captured by the single event $C S_{p}$, so stat ${ }_{p}$ changes directly from entry to exit.
} 


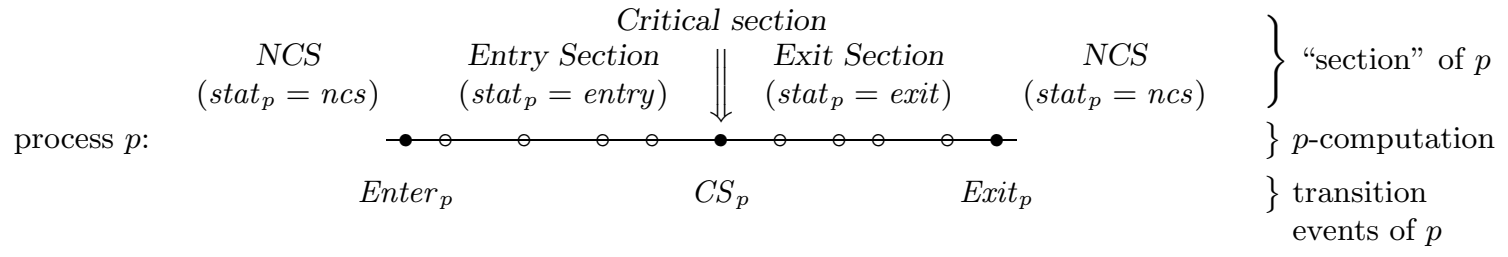

Figure 2: Transition events of an atomic mutual exclusion system. In this figure, NCS stands for "noncritical section," a circle (०) represents a non-transition event, and a bullet (•) represents a transition event.

In our proof, we only consider computations in which each process enters and then exits its critical section at most once. Thus, we henceforth assume that each computation contains at most one Enter ${ }_{p}$ event for each

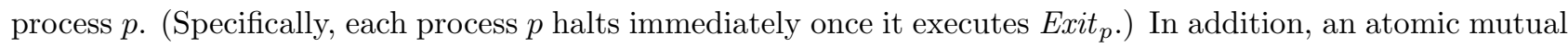
exclusion system is required to satisfy the following.

Exclusion: For all $H \in C$, if both $H \circ\left\langle C S_{p}\right\rangle \in C$ and $H \circ\left\langle C S_{q}\right\rangle \in C$ hold, then $p=q$.

Progress: Given $H \in C$, define $X=\left\{q \in P \mid\right.$ value $\left.\left._{\text {stat }}, H\right) \neq n c s\right\}$. If $X$ is nonempty, then there exists an $X$-computation $G$ and a process $p \in X$ such that $H \circ G \circ\left\langle e_{p}\right\rangle \in C$ and $e_{p}$ is either $C S_{p}$ (if value $\left(\right.$ stat $\left._{p}, H\right)=$

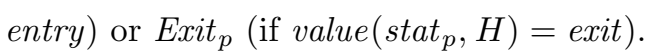

The Exclusion property precludes multiple critical-section events from being simultaneously "enabled." Although we assume that each critical-section execution is vacuous, we can certainly "augment" a given algorithm by replacing each event $C S_{p}$ by a set of events that represents $p$ 's critical-section execution. If two events $C S_{p}$ and $C S_{q}$ are simultaneously enabled after a computation $H$, then we can interleave the critical-section executions of $p$ and $q$, thus violating mutual exclusion. The Exclusion property states that such a situation does not arise.

The Progress property is implied by livelock-freedom, although it is strictly weaker than livelock-freedom. In particular, it allows the possibility of infinitely extending $H$ such that no active process $p$ executes $C S_{p}$ or Exit $_{p}$. This weaker formalism is sufficient for our purposes.

\subsection{Cache-Coherent Systems}

On cache-coherent (CC) shared-memory systems, some remote-variable accesses may be handled locally, without causing interconnection network traffic. Our lower-bound proof applies to such systems without modification. This is because we do not count every remote event, but only certain "critical" events that generate cache misses. (Actually, as explained below, some events that we consider critical might not generate cache misses in certain system implementations, but this has no asymptotic impact on our proof.) The notion of a critical event presented here is taken directly from [4].

Precisely defining the class of such events in a way that is applicable to the myriad of cache implementations that exist is exceedingly difficult. We partially circumvent this problem by assuming idealized caches of infinite size: a cached variable may be updated or invalidated but it is never replaced by another variable. Note that, in practice, cache size and associativity limitations should only increase the number of cache misses. In addition, in order to keep the proof manageable, we allow cache misses to be both undercounted and overcounted. In particular, as explained below, in any realistic cache system, at least a constant fraction (but not necessarily all) of all critical events generate cache misses. Thus, a single cache miss may be associated with $\Theta(1)$ critical events, resulting in overcounting up to a constant factor. Note that this overcounting has no effect on our asymptotic lower bound. Also, an event that generates a cache miss may be considered noncritical, resulting in 
undercounting, which may be of more than a constant factor. Note that this undercounting can only strengthen our asymptotic lower bound. Therefore, an asymptotic lower bound on the number of critical events is also an asymptotic lower bound on the number of actual cache misses.

Our definition of a critical event is given below. This definition is followed by a rather detailed explanation in which various kinds of caching protocols are considered.

Definition: Let $\mathcal{S}=(C, P, V)$ be an atomic mutual exclusion system. Let $e_{p}$ be an event in $H \in C$. Then, we can write $H$ as $F \circ\left\langle e_{p}\right\rangle \circ G$, where $F$ and $G$ are subcomputations of $H$. We say that $e_{p}$ is a critical event in $H$ if and only if one of the following conditions holds:

Transition event: $e_{p}$ is one of Enter,$C S_{p}$, or Exit .

Critical read: There exists a variable $v$, remote to $p$, such that $o p\left(e_{p}\right)=\operatorname{read}(v)$ and $F \mid p$ does not contain a read from $v$.

- Informally, $e_{p}$ is the first event of $p$ that reads $v$ in $H$.

Critical write: There exists a variable $v$, remote to $p$, such that $e_{p}$ is a remote write event on $v$ (i.e., $\left.o p\left(e_{p}\right)=\operatorname{write}(v)\right)$, and writer $(v, F) \neq p$.

- Informally, $e_{p}$ is a remote write event on $v$, and either $e_{p}$ is the first event that writes to $v$ in $H$ (i.e., writer $(v, F)=\perp$ ), or $e_{p}$ overwrites a value that was written by another process.

Critical successful comparison: There exists a variable $v$, remote to $p$, such that $e_{p}$ is a successful comparison event on $v$ (i.e., op $\left(e_{p}\right)=\operatorname{compare}(v, \alpha)$ for some value of $\alpha$ and $\left.v \in \operatorname{Wvar}\left(e_{p}\right)\right)$, and $\operatorname{writer}(v, F) \neq p$. - Informally, $e_{p}$ is a successful comparison event on $v$, and either $e_{p}$ is the first event that writes to $v$ in $H$ (i.e., writer $(v, F)=\perp$ ), or $e_{p}$ overwrites a value that was written by another process.

Critical unsuccessful comparison: There exists a variable $v$, remote to $p$, such that $e_{p}$ is an unsuccessful comparison event on $v\left(i . e .\right.$, op $\left(e_{p}\right)=\operatorname{compare}(v, \alpha)$ for some value of $\alpha$ and $\left.v \notin \operatorname{Wvar}\left(e_{p}\right)\right)$, writer $(v, F) \neq$ $p$, and either

(i) $F \mid p$ does not contain an unsuccessful comparison event on $v$, or

(ii) $F$ can be written as $F_{1} \circ\left\langle f_{q}\right\rangle \circ F_{2}$, where $q \neq p$ and $f_{q}=$ writer_event $(v, F)$, such that $F_{2} \mid p$ does not contain an unsuccessful comparison event on $v$.

- Informally, $e_{p}$ must read the initial value of $v$ (if $\operatorname{writer}(v, F)=\perp$ ) or a value that is written by another process q. Moreover, either (i) $e_{p}$ is the first unsuccessful comparison on $v$ by $p$ in $H$, or (ii) $e_{p}$ is the first such event by $p$ after some other process has written to $v\left(\right.$ via $\left.f_{q}\right) .^{9}$

Note that state transition events do not actually cause cache misses; these events are defined as critical so that certain cases can be combined in the proofs that follow. A process executes only three transition events per critical-section execution, so defining transition events as critical does not affect our asymptotic lower bound.

It is possible that the first read of $v$ by $p$, the first write or successful comparison event on $v$ by $p$, and the first unsuccessful comparison event on $v$ by $p$ (i.e., Case (i) in the definition above) are all considered critical. Depending on the system implementation, the second and third of these events (in the order of occurrence) might not generate a cache miss. However, even in such a case, the first such event will always generate a cache miss, and hence at least a third of all such "first" critical events will actually incur real interconnect traffic. Hence, considering all of these events to be critical has no asymptotic impact on our lower bound.

\footnotetext{
${ }^{9}$ This definition is more complicated than those for critical writes and successful comparisons because an unsuccessful comparison event on $v$ by $p$ does not actually write $v$. Thus, if a sequence of such events is performed by $p$ while $v$ is not written by other processes, then only the first such event should be considered critical.
} 
All caching protocols are based on either a write-through or a write-back scheme. In a write-through scheme, all writes go directly to shared memory. In a write-back scheme, a remote write to a variable $v$ may create a cached copy of $v$, so that subsequent writes to $v$ do not cause cache misses. With either scheme, if cached copies of $v$ exist on other processors when such a write occurs, then to ensure consistency, these cached copies must be either invalidated or updated. In the rest of this subsection, we consider in some detail the question of whether our notion of a critical write and a critical comparison is reasonable under the various caching protocols that arise from these definitions.

First, consider a system in which there are no comparison events, in which case it is enough to consider only critical write events. If a write-through scheme is used, then all remote write events cause interconnect traffic, so consider a write-back scheme. In this case, a write $e_{p}$ to a remote variable that is not the first write to $v$ by $p$ is considered critical only if $\operatorname{writer}(v, F)=q$ holds for some $q \neq p$, which implies that $v$ is stored in a local cache line of process $q$. In such a case, $e_{p}$ must either invalidate or update the cached copy of $v$ (depending on the means for ensuring consistency), thereby generating interconnect traffic.

Next, consider comparison events. A successful comparison event on a remote variable $v$ writes a new value to $v$. Thus, the reasoning given above for ordinary writes applies to successful comparison events as well. This leaves only unsuccessful comparison events. Recall that an unsuccessful comparison event on a remote variable $v$ does not actually write $v$. Thus, the reasoning above does not apply to such events.

In the remainder of this discussion, let $e_{p}$ denote an unsuccessful comparison event on a remote variable $v$, where Case (ii) in the definition applies. Then, some other process $q$ writes to $v$ (via a write or successful comparison event, or even a local, read, or unsuccessful comparison event, if $v$ is local to $q$ ) prior to $e_{p}$ but after $p$ 's most recent unsuccessful comparison event on $v$, and also after $p$ 's most recent successful comparison and/or remote write event on $v$. Consider the interconnect traffic generated, assuming an invalidation scheme for ensuring cache consistency. In this case, $p$ 's previous cached copy of $v$ is invalidated prior to $e_{p}$, so $e_{p}$ must generate interconnect traffic in order to read the current value of $v$, unless an earlier read of $v$ by $p$ (after $q$ 's write) exists. Thus, $e_{p}$ fails to generate interconnect traffic only if there is an earlier read of $v$ by $p$ (after $q$ 's write), say $f_{p}$, that does. Note that $f_{p}$ is either a "first" read of $v$ by $p$ or a noncritical read. The former case may happen at most once per remote variable; in the latter case, we can "charge" the interconnect traffic generated by $f_{p}$ to $e_{p}$.

The last possibility to consider is that of an unsuccessful comparison event $e_{p}$ implemented within a caching protocol that uses updates to ensure consistency. In this case, $q$ 's write in the scenario above updates $p$ 's cached copy, and hence $e_{p}$ may not generate interconnect traffic. (Note that, for interconnect traffic to be avoided in this case, the hardware must be able to distinguish a failed comparison event on a cached variable from a successful comparison event or a failed comparison on a non-cached variable.) Therefore, our lower bound does not apply to a system that uses updates to ensure consistency and that has the ability to execute failed comparison events on cached variables without generating interconnect traffic. (If an update scheme is used, but the hardware is incapable of avoiding interconnect traffic when executing such failed comparison events, then our lower bound obviously still applies.) In fact, an algorithm with $O(1)$ time complexity in such systems is presented in [4].

As a final comment on our notion of a critical event, notice that whether an event is considered critical depends on the particular computation that contains the event, specifically the prefix of the computation preceding the event. Therefore, when saying that an event is (or is not) critical, the computation containing the event must be specified. 


\section{Proof Strategy}

In Section 4, we show that for any positive $k$, there exists some $\bar{N}$ such that, for any mutual exclusion system $\mathcal{S}=(C, P, V)$ with $|P| \geq \bar{N}$, there exists a computation $H$ such that some process $p$ experiences point contention $k$ and executes at least $k$ critical events to enter and exit its critical section. In this section, we sketch the key ideas of the proof.

\subsection{Process Groups and Regular Computations}

Our proof focuses on a special class of computations called "regular" computations. The $\Omega(\log N / \log \log N)$ lower bound mentioned earlier was also proved by considering such computations, so most of the definitions in this subsection are taken directly from [4]. A regular computation consists of events of two groups of processes, "active processes" and "finished processes." Informally, an active process is a process in its entry section, ${ }^{10}$ competing with other active processes; a finished process is a process that has executed its critical section once, and is in its noncritical section. (Recall that we consider only computations in which each process executes its critical section at most once.) The allowed states of these processes are formally defined in Condition RF4, given later in this section.

Definition: Let $\mathcal{S}=(C, P, V)$ be a mutual exclusion system, and $H$ be a computation in $C$. We define $\operatorname{Act}(H)$, the set of active processes in $H$, and $\operatorname{Fin}(H)$, the set of finished processes in $H$, as follows.

$$
\begin{aligned}
& \operatorname{Act}(H)=\left\{p \in P|H| p \neq\langle\rangle \text { and }\left\langle\text { Exit }_{p}\right\rangle \text { is not in } H\right\} \\
& \operatorname{Fin}(H)=\left\{p \in P|H| p \neq\langle\rangle \text { and }\left\langle\text { Exit }_{p}\right\rangle \text { is in } H\right\}
\end{aligned}
$$

Initially, we start with a regular computation in which all the processes in $P$ are active. The proof proceeds by inductively constructing longer and longer regular computations, until the desired lower bound is attained. The regularity condition defined below ensures that no participating process has "knowledge" of any other process that is active. ${ }^{11}$ This has two consequences: we can "erase" any active process (i.e., remove its events from the computation) and still get a valid computation; "most" active processes have a "next" non-transition critical event. In each induction step, we append to each of the $n$ active processes (except at most one) one next critical event. These next critical events may introduce unwanted information flow, i.e., these events may cause an active process to acquire knowledge of another active process, resulting in a non-regular computation. Informally, such information flow is problematic because an active process $p$ that learns of another active process may start busy waiting. If $p$ busy waits via a local spin loop, then it might not execute any more critical events, in which case the induction fails.

In some cases, we can eliminate all information flow by simply erasing some active processes. Sometimes erasing alone does not leave enough active processes for the next induction step. In this case, we partition the active processes into two categories: "invisible" processes and "promoted" processes. The invisible processes (that are not erased - see below) will constitute the set of active processes for the next regular computation in the induction. No process is allowed to have knowledge of another process that is invisible. The promoted processes are processes that have been selected to "roll forward." A process that is rolled forward finishes executing its entry, critical, and exit sections, and returns to its noncritical section. (Both of these techniques,

\footnotetext{
${ }^{10}$ In our proof, some non-regular computations al so appear as intermediate steps between regular computations. In these nonregular computations, an active process may also be in its exit section.

${ }^{11} \mathrm{~A}$ process $p$ has knowledge of another process $q$ if $p$ has read from some variable a value that is written either by $q$ or another process that has knowledge of $q$.
} 


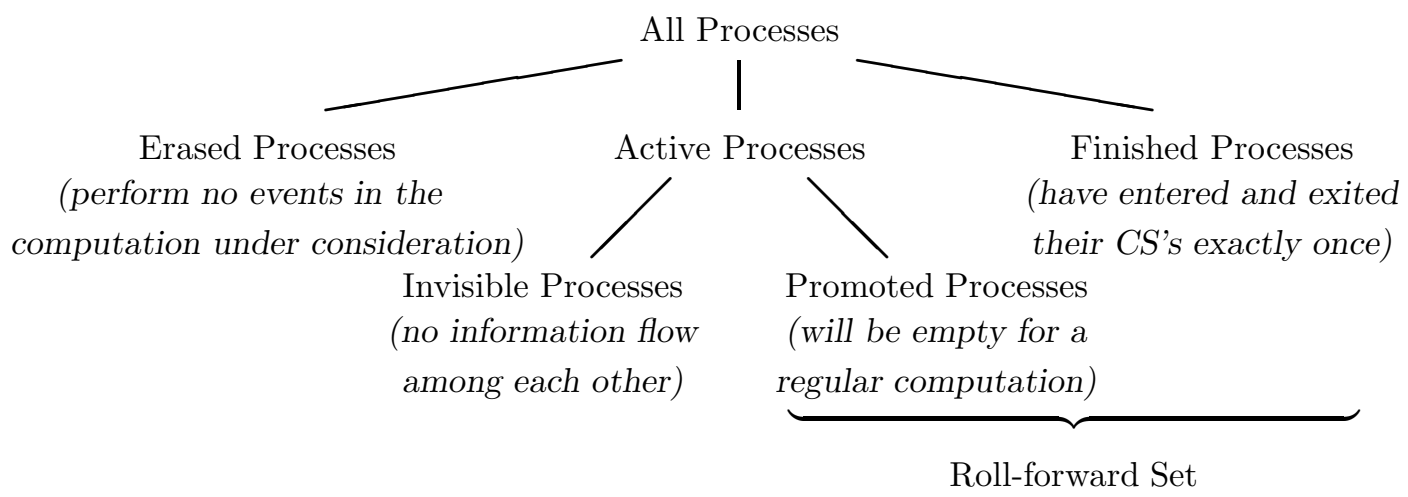

Figure 3: Process groups.

erasing and rolling forward, have been used previously to prove other lower bounds related to the mutual exclusion problem $[4,6,13,14,16]$, as well as several other lower bounds for concurrent systems [2, 36].) Processes are allowed to have knowledge of promoted or finished processes. Although invisible processes may have knowledge of promoted processes, once all promoted processes have finished execution, the regularity condition holds again (i.e., all active processes are invisible). The various process groups we consider are depicted in Figure 3 (the roll-forward set is discussed below).

The promoted and finished processes together constitute a "roll-forward set," which must meet Conditions RF1-RF5 below. Informally, Condition RF1 ensures that an invisible process is not known to any other processes. Conditions RF2 and RF3 bound the number of possible conflicts caused by appending a critical event. In particular, RF2 prevents information flow between two invisible processes via a variable local to either of them; RF3 prevents the erasing of an invisible process $p$ from "uncovering" a write by some other process that was subsequently overwritten by $p$. (For example, assume that RF3 is violated and that processes $q$ and $p$, both invisible, are the penultimate and the last writer to $v$, respectively. We may later want to let some process $r$ read $v$, as a means of obtaining a longer computation. In order to eliminate information flow, we have to erase both $p$ and $q$, because erasing $p$ alone allows $r$ to obtain knowledge of $q$. In general, we may have to erase an unbounded number of processes, which makes it rather difficult to proceed with the proof.) Condition RF4 ensures that the invisible, promoted, and finished processes behave as explained above. Condition RF5 ensures that we can erase any invisible process, maintaining that critical events (that are not erased) remain critical.

Definition: Let $\mathcal{S}=(C, P, V)$ be a mutual exclusion system, $H$ be a computation in $C$, and $R F S$ be a subset of $P$ such that $\operatorname{Fin}(H) \subseteq R F S$ and $H \mid p \neq\langle\rangle$ for each $p \in R F S$. We say that RFS is a valid roll-forward set (RF-set) of $H$ if and only if the following conditions hold.

RF1: Assume that $H$ can be written as $E \circ\left\langle e_{p}\right\rangle \circ F \circ\left\langle f_{q}\right\rangle \circ G .^{12}$ If $p \neq q$ and there exists a variable $v \in W v a r\left(e_{p}\right) \cap \operatorname{Rvar}\left(f_{q}\right)$ such that $F$ does not contain a write to $v$ (i.e., writer_event $\left.(v, F)=\perp\right)$, then $p \in R F S$ holds.

- Informally, if a process $p$ writes to a variable $v$, and if another process $q$ reads that value from $v$ without any intervening write to $v$, then $p \in R F S$ holds.

RF2: For any event $e_{p}$ in $H$ and any variable $v$ in $\operatorname{var}\left(e_{p}\right)$, if $v$ is local to another process $q(\neq p)$, then either $q \notin \operatorname{Act}(H)$ or $\{p, q\} \subseteq R F S$ holds.

\footnotetext{
${ }^{12}$ Here and in similar sentences hereafter, we are considering every way in which $H$ can be so decomposed. That is, any pair of events $e_{p}$ and $f_{q}$ inside $H$ such that $e_{p}$ comes before $f_{q}$ defines a decomposition of $H$ into $E \circ\left\langle e_{p}\right\rangle \circ F \circ\left\langle f_{q}\right\rangle \circ G$, and RF1 must hold for any such decomposition.
} 
- Informally, if a process $p$ accesses a variable that is local to another process $q$, then either $q$ is not an active process in $H$, or both $p$ and $q$ belong to the roll-forward set RFS. Note that this condition does not distinguish whether $q$ actually accesses $v$ or not, and conservatively requires $q$ to be in RFS (or erased) even if $q$ does not access $v$. This is done in order to simplify bookkeeping.

RF3: Suppose there is a variable $v \in V$ and two different events $e_{p}$ and $f_{q}$ in $H$ such that $p \neq q$, both $p$ and $q$ are in $\operatorname{Act}(H), v \in \operatorname{var}\left(e_{p}\right) \cap \operatorname{var}\left(f_{q}\right)$, and there exists a write to $v$ in $H$. Then, writer $(v, H) \in R F S$ holds. - Informally, if a variable $v$ is accessed by more than one process in $\operatorname{Act}(H)$, then the last process in $H$ to write to $v$ (if any) belongs to RFS.

RF4: For any process $p$ such that $H \mid p \neq\langle\rangle$,

$$
\text { value }\left(\text { stat }_{p}, H\right)= \begin{cases}\text { entry } & \text { if } p \in \operatorname{Act}(H)-R F S, \\ \text { entry or exit } & \text { if } p \in \operatorname{Act}(H) \cap R F S, \\ n c s & \text { otherwise }(i . e ., p \in \operatorname{Fin}(H)) .\end{cases}
$$

Moreover, if $p \in \operatorname{Fin}(H)$, then the last event by $p$ in $H$ is Exit $p$.

- Informally, if a process $p$ participates in $H(H \mid p \neq\langle\rangle)$, then at the end of $H$, one of the following holds: (i) $p$ is in its entry section and has not yet executed its critical section $(p \in \operatorname{Act}(H)-R F S)$; (ii) $p$ is in the process of "rolling forward" and is in its entry or exit section $(p \in \operatorname{Act}(H) \cap R F S)$; or (iii) $p$ has already finished its execution and is in its noncritical section (i.e., $p \in \operatorname{Fin}(H))$. Note that $\operatorname{Fin}(H)=R F S-\operatorname{Act}(H)$.

RF5: For any event $e_{p}$ in $H$, if $e_{p}$ is a critical write or a critical comparison in $H$, then $e_{p}$ is also a critical write or a critical comparison in $H \mid(\{p\} \cup R F S)$.

- Informally, if an event $e_{p}$ in $H$ is a critical write or a critical comparison, then it remains critical if we erase all processes not in RFS and different from $p$.

Condition RF5 is used to show that the property of being a critical write/comparison is conserved when considering certain related computations. Recall that, if $e_{p}$ is not the first event by $p$ to write to $v$, then for it to be critical, there must be a write to $v$ by another process $q$ in the subcomputation between $p$ 's most recent write (via a remote write or a successful comparison event) and event $e_{p}$. Similarly, if $e_{p}$ is not the first unsuccessful comparison by $p$ on $v$, then for it to be critical, there must be a write to $v$ by another process $q$ in the subcomputation between $p$ 's most recent unsuccessful comparison on $v$ and event $e_{p}$. RF5 ensures that if $q$ is not in RFS, then some other process $q^{\prime}$ exists that is in RFS and that writes to $v$ in the subcomputation in question.

Note that a valid RF-set can be "expanded": if $R F S$ is a valid RF-set of computation $H$, then any set of processes that participate in $H$, provided that it is a superset of $R F S$, is also a valid RF-set of $H$. Also note that $\operatorname{Act}(H) \cup \operatorname{Fin}(H)$ (the set of all participating processes) is always a valid RF-set, since we assume that each process $p$ halts as soon as it executes Exitp (see Section 2.2).

The invisible and promoted processes (which partition the set of active processes) are defined as follows.

Definition: Let $\mathcal{S}=(C, P, V)$ be a mutual exclusion system, $H$ be a computation in $C$, and $R F S$ be a valid RF-set of $H$. We define $\operatorname{Inv}_{R F S}(H)$, the set of invisible processes in $H$, and $\operatorname{Pmt}_{R F S}(H)$, the set of promoted processes in $H$, as follows.

$$
\begin{aligned}
\operatorname{Inv}_{R F S}(H) & =\operatorname{Act}(H)-R F S \\
\operatorname{Pmt}_{R F S}(H) & =\operatorname{Act}(H) \cap R F S
\end{aligned}
$$


For brevity, we often omit the specific RF-set if it is obvious from the context, and simply use the notation $\operatorname{Inv}(H)$ and $\operatorname{Pmt}(H)$. Finally, the regularity condition can be defined as "all the processes we wish to roll forward have finished execution."

Definition: A computation $H$ in $C$ is regular if and only if $\operatorname{Fin}(H)$ is a valid RF-set of $H$.

\subsection{Detailed Proof Overview}

Initially, we start with a regular computation $H_{1}$, where $\operatorname{Act}\left(H_{1}\right)=P, \operatorname{Fin}\left(H_{1}\right)=\{\}$, and each process has one

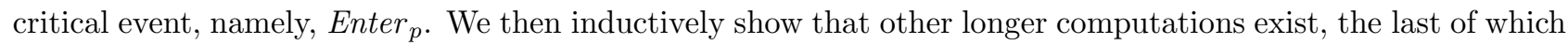
establishes our lower bound. Each computation is obtained by rolling forward or erasing some processes. The induction is complete when we obtain $H_{k}$; throughout the rest of this section, $k$ represents a given fixed number. We assume that $P$ is large enough to ensure that enough non-erased processes remain after each induction step for the next step to be applied. The precise bound on $|P|$, as a function of $k$, is given in Theorem 2 .

At the $j^{\text {th }}$ induction step, we consider a regular computation $H_{j}$ such that $\operatorname{Act}\left(H_{j}\right)$ consists of $n$ processes that execute $j$ critical events each (in $H_{j}$ ). We construct a regular computation $H_{j+1}$ such that

- $\operatorname{Act}\left(H_{j+1}\right)$ consists of $\Omega(\sqrt{n} / k)$ processes, and

- each active process in $\operatorname{Act}\left(H_{j+1}\right)$ executes $j+1$ critical events in $H_{j+1}$.

The construction method, formally described in Lemma 7, is explained below. In constructing $H_{j+1}$ from $H_{j}$, we may erase some processes and roll at most two processes forward (thereby making them finished processes). After executing steps $1, \ldots,(k-1)$, we have a regular computation $H_{k}$ in which each active process executes $k$ critical events and $\left|\operatorname{Fin}\left(H_{k}\right)\right| \leq 2(k-1)$. Since active processes have no knowledge of each other, we may erase all but one active process from $H_{k}$ and obtain a valid computation. This computation has exactly one active process and at most $2(k-1)$ finished processes. Thus, its contention is at most $2 k-1$. Moreover, the single remaining active process performs $k$ critical events, proving the desired lower bound.

We now describe how $H_{j+1}$ is constructed from $H_{j}$. Let $n=\left|\operatorname{Act}\left(H_{j}\right)\right|$. Since $H_{j}$ is regular, no active process has knowledge of other active processes. Therefore, we can "erase" any active process and still get a valid computation (Lemma 1). Moreover, if we choose an active process $p$ and erase all other active processes, then by the Progress property, $p$ eventually executes $C S_{p}$. We claim that every process $p$ in $\operatorname{Act}\left(H_{j}\right)$, except at most one, executes at least one additional critical event before it executes $C S_{p}$. This claim is formally stated and proved in Lemma 5; here we give an informal explanation.

Assume, for the sake of contradiction, that we have two distinct active processes, $p$ and $q$, each of which may execute its $C S$ event, if executed alone, by first executing only noncritical events. That is, we have two valid computations $H_{j} \circ L_{p} \circ\left\langle C S_{p}\right\rangle$ and $H_{j} \circ L_{q} \circ\left\langle C S_{q}\right\rangle$, where $L_{p}$ and $L_{q}$ consist solely of noncritical events. It can be shown that noncritical events of invisible processes cannot cause any information flow among these processes (Lemma 4). Hence, we can append both $L_{p}$ and $L_{q}$ after $H_{j}$ and still obtain a valid computation, as shown below.

Claim 1: $H_{j} \circ L_{p} \circ L_{q}$ is a valid computation.

Proof of Claim: It suffices to prove that $q$ cannot distinguish between $H_{j}$ and $H_{j} \circ L_{p}$. (From this, it follows that, if $q$ can execute $L_{q}$ after $H_{j}$, then it can do so after $H_{j} \circ L_{p}$.)

Consider each event $e_{q}$ in $L_{q}$ (in $H_{j} \circ L_{p} \circ L_{q}$ ). Event $e_{q}$ may distinguish between $H_{j}$ and $H_{j} \circ L_{p}$ only if it reads a variable (say, $v$ ) that is written by some event in $L_{p}$. Since events in $L_{p}$ are noncritical, 


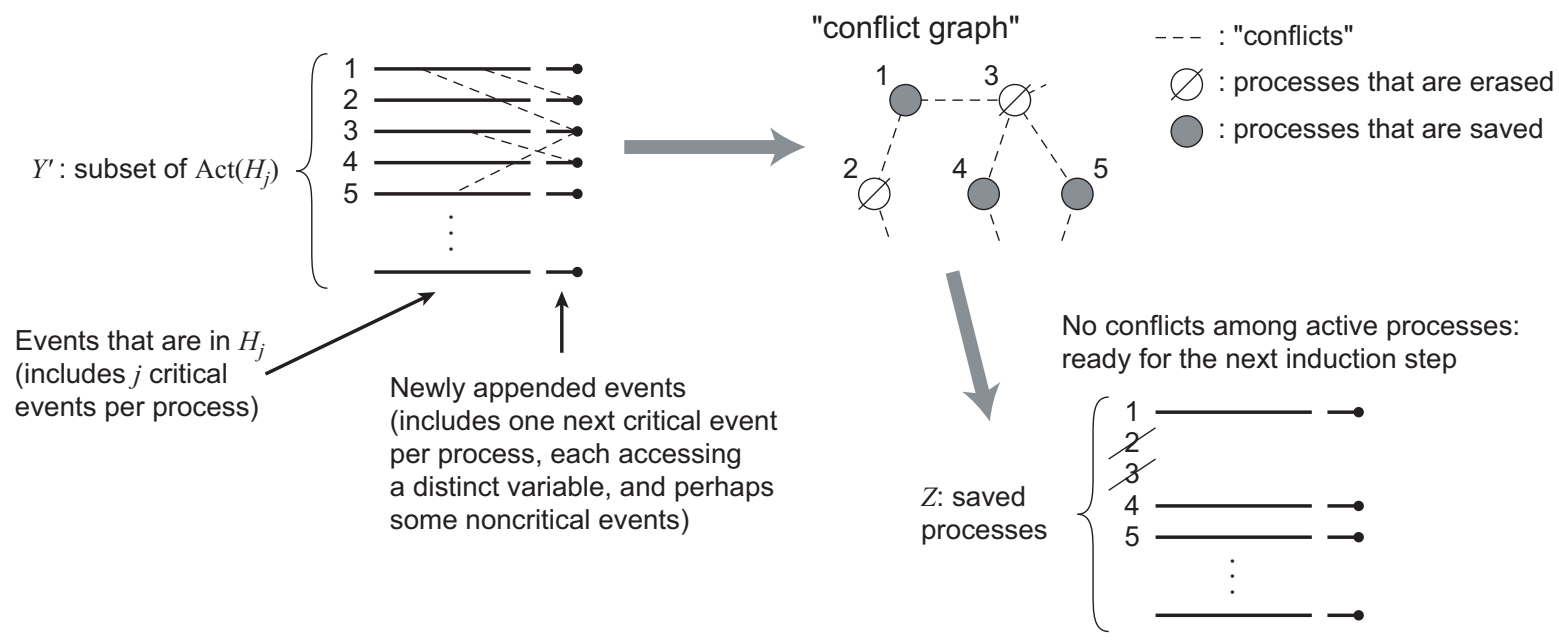

Figure 4: Erasing strategy. For simplicity, processes in $\operatorname{Fin}\left(H_{j}\right)$ are not shown.

either $v$ is local to $p$ or there exists a critical event $f_{p}$ in $H_{j}$ that writes $v$. Similarly, since $e_{q}$ is noncritical (in $H_{j} \circ L_{q}$ ), either $v$ is local to $q$ or there exists a critical event $g_{q}$ in $H_{j}$ that reads $v$.

If $v$ is local to $p$, then by applying RF2 to $H_{j}$ (with ' $R F S$ ' $\leftarrow \operatorname{Fin}\left(H_{j}\right)$, ' $p$ ' $\leftarrow q$, ' $q$ ' $\leftarrow p$, and ' $e_{p}$ ' $\left.\leftarrow g_{q}\right),{ }^{13}$ and using $\{p, q\} \subseteq \operatorname{Act}\left(H_{j}\right)$, we have a contradiction. If $v$ is local to $q$, then similarly applying RF2, we again have a contradiction. The only remaining possibility is that $v$ is remote to both $p$ and $q$, and that $H_{j}$ contains events $f_{p}$ (which writes $v$ ) and $g_{q}$ (which reads $v$ ). Then, by RF3, we have $\operatorname{writer}\left(v, H_{j}\right) \in \operatorname{Fin}\left(H_{j}\right)$. In particular, writer $\left(v, H_{j}\right)$ is not $q$. However, this implies that the first event (either $e_{q}$ or some earlier event) to read $v$ in $L_{q}$ (as a subcomputation of $H_{j} \circ L_{q}$ ) is critical, by definition. Hence, we have reached a contradiction.

Since $C S_{p}$ is a "dummy" event that does not read any variable, it cannot distinguish between $H_{j} \circ L_{p}$ and $H_{j} \circ L_{p} \circ L_{q}$. (Similar reasoning applies to $C S_{q}$.) It follows that $H_{j} \circ L_{p} \circ L_{q}$ may be followed by both $C S_{p}$ and $C S_{q}$, clearly violating the Exclusion property.

Thus, among the $n$ processes in $\operatorname{Act}\left(H_{j}\right)$, at least $n-1$ processes execute an additional critical event before entering their critical sections. We call these events "next" critical events, and denote the corresponding set of processes by $Y$. We consider two cases, based on the variables remotely accessed by these next critical events.

Erasing strategy. Assume that there are at least $\sqrt{n}$ distinct variables that are remotely accessed by some next critical events. For each such variable $v$, we select one process whose next critical event accesses $v$. Let $Y^{\prime}$ be the set of selected processes. This situation is depicted in Figure 4. We now eliminate remaining possible conflicts among processes in $Y^{\prime}$ by constructing a "conflict graph" $\mathcal{G}$ as follows. (Two processes conflict if the addition of new events by both processes creates information flow or violates one of RF1-RF5.)

Each process $p$ in $Y^{\prime}$ is considered a vertex in $\mathcal{G}$. By induction, process $p$ has $j$ critical events in $\operatorname{Act}\left(H_{j}\right)$ and one next critical event. For each of the $j+1$ critical events of $p$, (i) if the event accesses the same variable as the next critical event of some other process $q$, introduce edge $(p, q)$. In addition, (ii) if the next critical event of $p$ remotely accesses a local variable of $q$, also introduce edge $(p, q)$.

Since each process in $Y^{\prime}$ accesses a distinct remote variable in its next critical event, it is clear that each process generates at most $j+1$ edges by rule (i) and at most one edge by rule (ii). By applying Turán's theorem

\footnotetext{
${ }^{13}$ In the rest of this section, Conditions RF1-RF5 are applied to $H_{j}$ with 'RFS' $\leftarrow$ Fin $\left(H_{j}\right)$, unless specified otherwise.
} 
(Theorem 1, given later in Section 4), we can find a subset $Z$ of $Y^{\prime}$ such that $|Z|=\Omega(\sqrt{n} / j)$ and their critical events do not conflict with each other. (Since $H_{j}$ is regular, any conflict among critical events that are in $H_{j}$ must be already resolved. Thus, we can be sure that there are no remaining conflicts.) By retaining $Z$ and erasing all other active processes, we can eliminate all conflicts. We define the resulting computation as $H_{j+1}$, the desired computation.

To complete the proof, we still have to show that $H_{j+1}$ is a regular computation (satisfying RF1-RF5) and that each active process in $H_{j+1}$ (that is, each process in $Z$ ) executes $j+1$ critical events in $H_{j+1}$. Since a rigorous proof (given in Section 4) is mainly technical in nature and rather tedious to follow, here we only give a very cursory overview. Interested readers are referred to the proof of Lemma 7.

Lemmas 1 and 4 show that it is safe (i.e., both regularity and the "criticality" of each event are preserved) to erase active processes and append noncritical events, respectively. Hence, it suffices to consider only each next critical event (say, $e_{p}$ ) to be appended. Assume that $e_{p}$ accesses a variable $v$ remotely. ${ }^{14}$

Condition RF1 (with ' $e_{p}$ ' $\leftarrow f_{q}$ and ' $f_{q}$ ' $\leftarrow e_{p}$ ) can be violated only if $e_{p}$ reads from $v$ a value written by $f_{q}$, where $q$ is another active process. In this case, by rule (ii) (in defining edges), $v$ is remote to $q$. (Otherwise, $q$ is already erased.) Hence, there exists some critical event by $q$ that writes $v$ in $H_{j}$. But then $\mathcal{G}$ contains edge $(p, q)$ by rule (i), a contradiction.

Rule (ii) ensures $e_{p}$ cannot violate RF2.

Rule (i) ensures that $v$ is different from every variable remotely accessed by any active process other than p. (Recall that the first access to each remote variable is critical. Hence, a process cannot access a remote variable $u$ at all if it does not access it critically.) Also, rule (ii) ensures that $v$ is not local to any active process. It follows that $e_{p}$ and $v$ cannot meet the antecedent of RF3, and hence appending $e_{p}$ vacuously preserves RF3.

Since every next critical event is a non-transition event, RF4 is clearly preserved.

In order to show that RF5 is preserved and that $p$ executes $j+1$ critical events in $H_{j+1}$, it suffices to show that $e_{p}$ remains critical in both $H_{j+1}$ and $H_{j+1} \mid(\{p\} \cup R F S)$. If $e_{p}$ is critical because it is the first event by $p$ to write/read $v$, then $e_{p}$ is clearly critical regardless of other processes' computations. Hence, assume that $e_{p}$ is critical after $H_{j}$ because some earlier event $f_{p}$ writes $v$ in $H_{j}$ and some other process $q$ writes $v$ in $H_{j}$, after $f_{p}$. In this case, by RF3, the last process to write $v$ (say, $r$ ) in $H_{j}$ is a finished process, and hence cannot be erased. Therefore, in $H_{j+1}, e_{p}$ still reads/overwrites a value written by $r$, and remains critical. Moreover, $e_{p}$ remains critical even if we erase all active processes except $p$ from $H_{j+1}$, thus satisfying RF5. (Formally, this reasoning is given by Lemma 2.)

Roll-forward strategy. Assume that the number of distinct variables that are remotely accessed by some next critical events is less than $\sqrt{n}$. This situation is depicted in Figure 5 . Since there are at least $n-1$ next critical events, there exists a variable $v$ that is remotely accessed by next critical events of at least $(n-1) / \sqrt{n}$ $(=\Theta(\sqrt{n}))$ processes. Let $Y_{v}$ be the set of these processes. (In the following discussion, we consider $v$ as fixed.) First, we retain $Y_{v}$ and erase all other active processes. Let the resulting computation be $H^{\prime}$. (Formally, $H^{\prime}=H \mid\left(Y_{v} \cup \operatorname{Fin}(H)\right)$, as defined in (113); other equations referred to below can also be found in the proof of Lemma 7.) Note that, if executed in arbitrary order, processes in $Y_{v}$ may gather knowledge of each other by accessing the same variable $v$. Hence, the crux of our strategy lies in limiting such information flow.

We achieve this by arranging the next critical events of $Y_{v}$ by placing write, comparison, and read events in

\footnotetext{
${ }^{14}$ In fact, as shown in the proof of Lemma 7, we append $e_{p}^{\prime}$, an event that is congruent to $e_{p}$. (See (96) and (97).) This is because $e_{p}$ (after $H_{j}$ ) may read from $v$ a value written by another active process $q$; in this case, $q$ is erased, and $p$ may now read a different value from $v$, executing the same statement but generating a different event. This detail is ignored here in order to simplify the proof overview.
} 

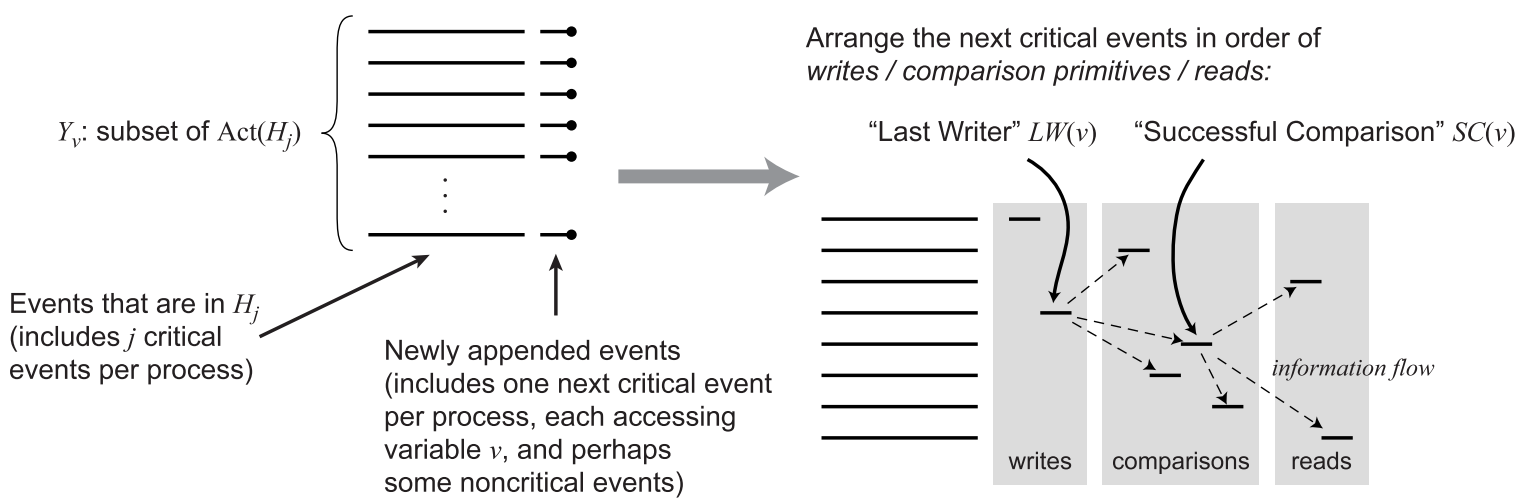

Figure 5: Roll-forward strategy. For simplicity, processes in Fin $\left(H_{j}\right)$ are not shown.

that order. In this way, all "next" write events (of $v$ ), except for the last one, are overwritten by subsequent writes, and hence cannot create any information flow. (That is, when some other process later reads $v$, it cannot gather any information of these "next" writers, except for the last one.) Furthermore, we can arrange comparison events such that at most one of them succeeds, as follows.

Let $\alpha$ be the value of $v$ after all the next write events are executed. We first append all comparison events that compare $v$ to any value different from $\alpha$, i.e., with an operation that can be written as compare $(v, \beta)$ such that $\beta \neq \alpha$. These comparison events must fail. We then append the remaining comparison events, namely, events with operation compare $(v, \alpha)$. If there are such events, the first comparison event is successful (changes the value of $v$ ) and all subsequent comparison events must fail.

Thus, among the next events (that are not erased so far), the only information flow that arises is from the "last writer" event $L W(v)$ and from the "successful comparison" event $S C(v)$ to all other next comparison and read events of $v$. We use $p_{\mathrm{LW}}$ and $p_{\mathrm{SC}}$ to denote the processes that execute $L W(v)$ and $S C(v)$, respectively. (Depending on the computation, we may have only one of them, or neither.)

As a concrete example, assume that $Y_{v}$ consists of eight active processes $p_{1}-p_{8}$ that are about to execute (as their next critical events) statements $s 1-s 8$, shown below. Also assume that $v$ equals 0 before the execution of these statements.

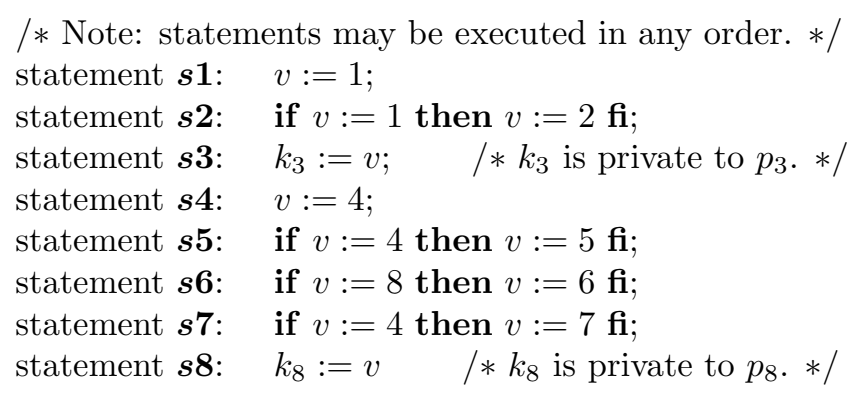

In this case, we may schedule these statements in the following order: $s 1, s 4$ ("last writer"), $s 2, s 6, s 5$ (the only successful comparison), $s 7, s 3$, and $s 8$. (This situation is depicted in the right side of Figure 5.) It is easy to see that the only information flow is from $p_{4}\left(=p_{\mathrm{LW}}\right)$ and $p_{5}\left(=p_{\mathrm{SC}}\right)$, that is, only $p_{4}$ and $p_{5}$ become visible.

We have thus constructed a non-regular computation, as $p_{\mathrm{LW}}$ and $p_{\mathrm{SC}}$ are active yet visible. (That is, $\operatorname{Fin}\left(H^{\prime}\right) \cup\left\{p_{\mathrm{LW}}, p_{\mathrm{SC}}\right\}$ is a valid roll-forward set.) We then roll $p_{\mathrm{LW}}$ and $p_{\mathrm{SC}}$ forward ${ }^{15}$ (i.e., allow them to execute their remaining entry/critical/exit sections, while stalling other active processes), thus generating a 
regular computation $G$ such that $\operatorname{Fin}(G)=\operatorname{Fin}\left(H^{\prime}\right) \cup\left\{p_{\mathrm{LW}}, p_{\mathrm{SC}}\right\}$. Toward this goal, we inductively construct a series of non-regular computations $G_{0}, G_{1}, \ldots, G_{l}(=G)$ such that exactly one of $p_{\mathrm{LW}}$ and $p_{\mathrm{SC}}$ executes one more critical event in $G_{h+1}$ than $G_{h}$. This additional critical event may cause $p_{\mathrm{LW}}$ or $p_{\mathrm{SC}}$ to read from a variable written by another active process, in which case we erase that single active process to prevent information flow. (See the induction following (155)-(161) for details.) The induction stops (at some step $l$ ) when both $p_{\mathrm{LW}}$ and $p_{\mathrm{SC}}$ transit to their noncritical sections, at which point we have erased at most $l$ active processes. The resulting computation $G_{l}$ is regular again, and this is the desired computation, $H_{j+1}$.

Having explained the crucial steps, we now give a more detailed explanation. Each process $p$ in $Y_{v}$ may execute some noncritical events before critically accessing $v$. That is, we have a valid computation $H \circ L_{p} \circ\left\langle e_{p}\right\rangle$, where $L_{p}$ consists entirely of noncritical events by $p$, and $e_{p}$ accesses $v$ critically. (See (59)-(64).) By definition, we can erase any subset of invisible processes and still obtain a valid computation. In particular, $H^{\prime} \circ L_{p} \circ\left\langle e_{p}\right\rangle$ is a valid computation (where $H^{\prime}=H \mid\left(Y_{v} \cup \operatorname{Fin}(H)\right)$ and we have erased $\left.\operatorname{Act}(H)-Y_{v}\right)$.

Lemma 4 shows that noncritical events of invisible processes cannot cause information flow. (We proved this for two processes in Claim 1; the argument can be easily generalized to an arbitrary number of processes.) Hence, $H^{\prime} \circ L$ is a valid computation, where $L$ is obtained by concatenating computations $L_{p}$ for each $p \in Y_{v}$.

By Property P3, each process $p$ in $Y_{v}$ must execute, after $H^{\prime} \circ L$, some event congruent to $e_{p}$. Thus we can obtain a computation $\bar{G}=H^{\prime} \circ L \circ E$ (see (134)), where $E$ contains all "next critical events" by processes in $Y_{v}$, arranged such that the only information flow is from $p_{\mathrm{LW}}$ and $p_{\mathrm{SC}}$, as explained above. We denote events in $E$ by $e_{p}^{\prime}$ for each $p \in Y_{v}$. (Either or both of $p_{\mathrm{LW}}$ and $p_{\mathrm{SC}}$ may be missing, e.g., if all next critical events are reads from $v$ and $v$ was never written before. For simplicity, here we assume that they both exist.)

It can be shown that every $e_{p}^{\prime}$ in $E$ is critical. (See Claim 4 in the proof of Lemma 7.) Informally, this follows because $e_{p}$ is critical in $H^{\prime} \circ L_{p} \circ\left\langle e_{p}\right\rangle$, and because inserting more events executed by other processes cannot cause a critical event to become noncritical.

We now expand our "roll-forward set" to include $p_{\mathrm{LW}}$ and $p_{\mathrm{SC}}$, i.e., we define $\operatorname{RFS}(\bar{G})=\operatorname{Fin}(H) \cup\left\{p_{\mathrm{LW}}, p_{\mathrm{SC}}\right\}$. (In the rest of this section, all stated conditions are with respect to this $\operatorname{RFS}(\bar{G})$, so we will omit the operand and simply use $R F S$ to denote $R F S(\bar{G})$.) Before rolling $p_{\mathrm{LW}}$ and $p_{\mathrm{SC}}$ forward, we must first show that $R F S$ is a valid roll-forward set of $\bar{G}$. As noted after the definition of RF1-RF5, we can always expand a valid RF-set, and hence $R F S$ is a valid RF-set of $H$. Also, as explained in the description of erasing strategy, it is safe to erase active processes and append noncritical events. It follows that RFS is a valid RF-set of $H^{\prime} \circ L$. Hence, we only need to show that the addition of the "next critical events" (i.e., those in $E$ ), when applied to a computation that satisfies RF1-RF5, preserves these conditions.

Claim 2: $R F S$ is a valid RF-set of $\bar{G}=H^{\prime} \circ L \circ E$.

Proof of Claim: We consider each condition separately.

- RF1: Condition RF1 easily follows from our construction, since the only information flow is from $p_{\mathrm{LW}}$ and $p_{\mathrm{SC}}$, which are included in RFS.

- RF2: Since each active process (say, $p$ ) is in $Y_{v}, p$ accesses $v$ remotely (in $E$ ) by definition, and hence $v$ cannot be local to any active process. Hence, RF2 is preserved. (Note that $\operatorname{Act}(\bar{G})=Y_{v}$.)

- RF3: Consider two different events $f_{p}$ and $g_{q}$ in $H$, such that $p \neq q$, both $p$ and $q$ are in $\operatorname{Act}(\bar{G})$, there exists a variable $u$ accessed by both $f_{p}$ and $g_{q}$, and there exists a write to $u$ in

\footnotetext{
${ }^{15}$ In some cases, $L W(v)$ may be an event in $H_{j}$, if all next critical events by processes in $Y_{v}$ are comparison or read events. In such a case, we still define $p_{\mathrm{LW}}$ as the process that execute $L W(v)$, and roll it forward if it is an active process.
} 
$\bar{G}$. Our proof obligation is to show writer $(u, \bar{G}) \in R F S$.

If both $f_{p}$ and $g_{q}$ are inside $H^{\prime} \circ L$, then we have the desired condition, because RFS is a valid RF-set of $H^{\prime} \circ L$. So, without loss of generality, assume that $g_{q}$ is an event in E, i.e., $g_{q}=e_{q}^{\prime}$. If $u=v$, then writer $(u, \bar{G})$ is either $p_{\mathrm{SC}}$ or (if $p_{\mathrm{SC}}$ does not exist) $p_{\mathrm{LW}}$, both of which are in RFS. We now show that $u \neq v$ is in fact impossible: assume $u \neq v$, for the sake of contradiction. Since $g_{q}\left(=e_{q}^{\prime}\right)$ remotely accesses $v$, by the Atomicity property, $u$ is local to $q$. Thus, $f_{p}$ accesses $u$ remotely, and hence $f_{p}$ cannot be an event in $E$ (because $v$ is the only variable remotely accessed in $E$ ). It follows that $f_{p}$ is an event in $H^{\prime} \circ L$. By applying RF2 with ' $R F S$ ' $\leftarrow \operatorname{Fin}(H)$ to $f_{p}$ in $H^{\prime} \circ L$, we have $q \notin \operatorname{Act}\left(H^{\prime} \circ L\right)$, which contradicts our assumption that $q \in \operatorname{Act}(\bar{G})$.

- RF4: Since every next critical event is a non-transition event, RF4 is clearly preserved.

- RF5: We need to show that each $e_{p}^{\prime}$ in $E$ remains critical when all other processes in $Y_{v}$ are erased. Here, we only consider the case when $e_{p}^{\prime}$ is a critical unsuccessful comparison; other cases are similar. (For a formal proof, see the proof of Lemma 2 in [4], and Claim 4 in the proof of Lemma 7.)

If $e_{p}^{\prime}$ is the first unsuccessful comparison event on $v$ by $p$, then it clearly remains critical regardless of other processes' events. Otherwise, let $f_{q}$ be the last event to write $v$ before $e_{p}^{\prime}$. Then $q \neq p$, and $e_{p}^{\prime}$ does not execute an unsuccessful comparison event on $v$ after $f_{q}$. Since $e_{p}^{\prime}$ is not the first unsuccessful comparison event by $p$ on $v, p$ does execute one (or more) before $f_{q}$. Let $g_{p}$ be one such unsuccessful comparison event on $v$.

We claim that $q \notin Y_{v}$, i.e., $f_{q}$ is not erased by erasing $Y_{v}-\{p\}$. (Then it clearly follows that $e_{p}^{\prime}$ remains critical.) Assume otherwise, and let $\bar{G}=F_{1} \circ\left\langle e_{p}^{\prime}\right\rangle \circ F_{2}$. Then, RFS is a valid RF-set of $F_{1}$ by induction, and $q \in Y_{v}=\operatorname{Act}\left(F_{1}\right)$. Applying RF3 to $F_{1}$ with ' $e_{p}$ ' $\leftarrow g_{p}$ and ' $f_{q}$ ' $\leftarrow f_{q}$, we have $q=\operatorname{writer}\left(v, F_{1}\right) \in R F S$, a contradiction.

We have thus established that RFS is a valid RF-set of $\bar{G}$. We now "roll forward" the two processes $p_{\mathrm{LW}}$ and $p_{\mathrm{SC}}$ : that is, we inductively construct a series of computations $G_{0}(=\bar{G}), G_{1}, \ldots, G_{l}(=G)$, each satifying the following:

(i) either $p_{\mathrm{LW}}$ or $p_{\mathrm{SC}}$ (but not both) executes one more critical event in $G_{h+1}$ than $G_{h}$;

(ii) RFS is a valid RF-set of each $G_{h}$;

(iii) $p_{\mathrm{LW}}$ and $p_{\mathrm{SC}}$ have no knowledge of other active processes, except possibly for each other.

At each step, we start with $G_{h}$, constructed in the previous step. If both $p_{\mathrm{LW}}$ and $p_{\mathrm{SC}}$ has reached their noncritical section in $G_{h}$, then we let $l=h$ and the induction terminates. Otherwise, either $p_{\mathrm{LW}}$ or $p_{\mathrm{SC}}$ is in its entry or exit section. By the Progress property, we can append to $G_{h}$ noncritical events by $p_{\mathrm{LW}}$ and/or $p_{\mathrm{SC}}$ until at least one executes some critical event.

Let $f_{p}$ be the appended critical event (where $p$ is either $p_{\mathrm{SC}}$ or $p_{\mathrm{LW}}$ ). $f_{p}$ may pose a problem if either it reads some variable (say, $u$ ) that was last written by some process $q \in \operatorname{Inv}\left(G_{h}\right)$, or if it remotely accesses some variable $u$ that is local to some process $q \in \operatorname{Inv}\left(G_{h}\right)$. (The former creates information flow and breaks RF1; the latter breaks RF2.) In either case, we erase $q$ from $G_{h}$; the resulting computation is $G_{h+1}$, which clearly satisfies (i).

We now show that $G_{h+1}$ satisfies (ii). As in other similar cases, appending noncritical events to $G_{h}$ cannot falsify any of RF1-RF5. (Since such a case involves a rather mechanical proof, we refer the reader to the proof of Lemma 6 in [4].) Thus, we only have to show that appending $f_{p}$ does not falsify RF1-RF5. We write $G_{h+1}=\hat{G} \circ \hat{L} \circ\left\langle f_{p}\right\rangle$, where $\hat{G}$ is the result of erasing $q$ from $G_{h}$ and $\hat{L}$ consists of noncritical events by $p_{\mathrm{SC}}$ and/or $p_{\mathrm{LW}}$. 
- RF1: For the sake of contradiction, assume that RF1 is violated. That is, there exists a variable $w$ that is last written by some process $r \notin R F S$ and then subsequently read by $f_{p}$. Because $r$ was not erased when we added $f_{p}$, there must be another process $q=\operatorname{writer}\left(w, G_{h}\right)$ that was erased instead, where $q \in \operatorname{Inv}\left(G_{h}\right)$. However, since both $q$ and $r$ write $w$ in $G_{h}$, by applying RF3 to $G_{h}$, we have $\operatorname{writer}\left(w, G_{h}\right) \in R F S$, a contradiction.

- RF2: If $f_{p}$ accesses a variable $w$ that is local to another process $q$, then as explained above, either $q \in R F S$ or $q$ was already erased. Hence, RF2 is preserved.

- RF3: Assume the antecedent of RF3 with ' $H$ ' $\leftarrow G_{h+1},{ }^{\prime} v$ ' $\leftarrow w$ (for some variable $w$ ), ' $e_{p}$ ' $\leftarrow f_{p}$, and ' $f_{q}$ ' $\leftarrow g_{r}$ (for some event $g_{r}$ ). If $f_{p}$ writes $w$, then $\operatorname{writer}\left(w, G_{h+1}\right)=p \in R F S$, so RF3 is preserved. Thus, assume that $f_{p}$ reads (but does not write) $w$. If $\hat{G} \circ \hat{L}$ contains multiple active processes accessing $w$, then by applying RF3 to $\hat{G} \circ \hat{L}$, we have $\operatorname{writer}\left(w, G_{h+1}\right)=\operatorname{writer}(w, \hat{G} \circ \hat{L}) \in R F S$. On the other hand, if $\hat{G} \circ \hat{L}$ contains only one active process writing $w$ (and no other active process reading $w$ ), then that process is already erased, as explained above.

- RF4 and RF5: It is clear that RF4 is preserved. The proof for RF5 is similar to the proof of Claim 2. (In particular, the fact that RF3 holds for $\hat{G} \circ \hat{L}$ plays a crucial role here.)

The roll-forward induction finishes at $G_{l}$ when both $p_{\mathrm{SC}}$ and $p_{\mathrm{LW}}$ reach their noncritical sections, at which point we have $\operatorname{Fin}\left(G_{l}\right)=R F S$, and hence $G_{l}$ is regular and we can define $H_{j+1}=G_{l}$.

Finally, we have to show that $H_{j+1}$ satisfies (1), that is, it has $\Omega(\sqrt{n} / k)$ active processes. Since we erase at most one active process to obtain $G_{h+1}$ from each $G_{h}$, we have $\left|\operatorname{Act}\left(H_{j+1}\right)\right| \geq|\operatorname{Act}(\bar{G})|-l$. But $\operatorname{Act}(\bar{G})=Y_{v}$ and $Y_{v}$ contains $\Omega(\sqrt{n})$ processes by our assumption.

If $l \geq 2 k$, then either $p_{\mathrm{LW}}$ or $p_{\mathrm{SC}}$ executes $k$ or more critical events in $G_{l}$, in which case our $\Omega(k)$ lower bound easily follows without any further induction. Therefore, we may assume $l<2 k$, in which case (1) follows by assuming that $k \ll n$, which can be guaranteed by starting with a sufficiently large number of active processes in $H_{1}$. (That is, we require the total number of processes in our system $(=N)$ to be so large that we have $\omega(k)$ processes at each induction step $j$ for $1 \leq j \leq k$. The precise bound is given by Theorem 2.)

\section{Detailed Lower Bound Proof}

In this section, we establish our lower-bound theorem. Throughout this section, we assume the existence of a fixed mutual exclusion system $\mathcal{S}=(C, P, V)$. We begin by stating six lemmas concerning mutual exclusion systems as defined here that were proved previously (in particular, in the paper that establishes the $\Omega(\log N / \log \log N)$ lower bound mentioned earlier) [4]. These lemmas are also numbered as Lemmas 1-6 in [4].

According to Lemma 1, stated next, any invisible process can be safely "erased."

Lemma 1 Consider a computation $H$ and two sets of processes RFS and $Y$. Assume the following:

- $H \in C$;

- RFS is a valid RF-set of $H$;

- $R F S \subseteq Y$.

Then, the following hold: $H \mid Y \in C$; RFS is a valid RF-set of $H \mid Y$; an event e in $H \mid Y$ is a critical event if and only if it is also a critical event in $H$. 
The next lemma shows that the property of being a critical event is conserved across "similar" computations. Informally, if process $p$ cannot distinguish two computations $H$ and $H^{\prime}$, and if $p$ may execute a critical event $e_{p}$ after $H$, then it can also execute a critical event $e_{p}^{\prime}$ after $H^{\prime} \circ G$, where $G$ is a computation that does not contain any events by $p$. Moreover, if $G$ satisfies certain conditions, then $H^{\prime} \circ G \circ\left\langle e_{p}^{\prime}\right\rangle$ satisfies RF5, preserving the "criticalness" of $e_{p}^{\prime}$ across related computations.

Lemma 2 Consider three computations $H, H^{\prime}$, and $G$, a set of processes RFS, and two events $e_{p}$ and $e_{p}^{\prime}$ of a process $p$. Assume the following:

- $H \circ\left\langle e_{p}\right\rangle \in C$;

- $H^{\prime} \circ G \circ\left\langle e_{p}^{\prime}\right\rangle \in C$;

- RFS is a valid RF-set of $H$;

- RFS is a valid RF-set of $H^{\prime}$;

- $e_{p} \sim e_{p}^{\prime}$

- $p \in \operatorname{Act}(H)$;

- $H\left|(\{p\} \cup R F S)=H^{\prime}\right|(\{p\} \cup R F S)$;

- $G \mid p=\langle\rangle$;

- no events in $G$ write any of p's local variables;

- $e_{p}$ is critical in $H \circ\left\langle e_{p}\right\rangle$.

Then, $e_{p}^{\prime}$ is critical in $H^{\prime} \circ G \circ\left\langle e_{p}^{\prime}\right\rangle$. Moreover, if the following conditions are true,

(A) $H^{\prime} \circ G$ satisfies RF5;

(B) if $e_{p}$ is a comparison event on a variable $v$, and if $G$ contains a write to $v$, then $G \mid R F S$ also contains a write to $v$.

then $H^{\prime} \circ G \circ\left\langle e_{p}^{\prime}\right\rangle$ also satisfies RF5.

The next lemma provides means of appending an event $e_{p}$ of an active process, while maintaining RF1 and RF2. This lemma is used inductively in order to extend a computation with a valid RF-set. Specifically, (21) guarantees that RF2 is satisfied, and (22) forces any information flow to originate from a process in $R F S$, thus satisfying RF1. (Note that, if $q=\perp, q=p$, or $v_{\text {rem }} \notin R v a r\left(e_{p}\right)$ holds, then no information flow occurs.)

Lemma 3 Consider two computations $H$ and $G$, a set of processes RFS, and an event $e_{p}$ of a process p. Assume the following:

- $H \circ G \circ\left\langle e_{p}\right\rangle \in C$;

- RFS is a valid RF-set of $H$;

- $p \in \operatorname{Act}(H)$;

- $H \circ G$ satisfies RF1 and RF2;

- $G$ is an $\operatorname{Act}(H)$-computation;

- $G \mid p=\langle\rangle$;

- if $e_{p}$ remotely accesses a variable $v_{\text {rem }}$, then the following hold:

- if $v_{\text {rem }}$ is local to a process $q$, then either $q \notin \operatorname{Act}(H)$ or $\{p, q\} \subseteq R F S$, and

- if $q=$ writer $\left(v_{\mathrm{rem}}, H \circ G\right)$, then one of the following hold: $q=\perp, q=p, q \in R F S$, or $v_{\mathrm{rem}} \notin R v a r\left(e_{p}\right)$.

Then, $H \circ G \circ\left\langle e_{p}\right\rangle$ satisfies RF1 and RF2.

The next lemma gives us means for extending a computation by appending noncritical events. 
Lemma 4 Consider a computation $H$, a set of processes $R F S$, and another set of processes $Y=\left\{p_{1}, p_{2}, \ldots\right.$, $\left.p_{m}\right\}$. Assume the following:

- $H \in C$;

- RFS is a valid RF-set of $H$;

- $Y \subseteq \operatorname{Inv}_{R F S}(H)$;

- for each $p_{j}$ in $Y$, there exists a computation $L_{p_{j}}$, satisfying the following:

- $L_{p_{j}}$ is a $p_{j}$-computation;

- $H \circ L_{p_{j}} \in C$;

- $L_{p_{j}}$ has no critical events in $H \circ L_{p_{j}}$, that is, no event in $L_{p_{j}}$ is a critical event in $H \circ L_{p_{j}}$.

Define $L$ to be $L_{p_{1}} \circ L_{p_{2}} \circ \cdots \circ L_{p_{m}}$. Then, the following hold: $H \circ L \in C, R F S$ is a valid RF-set of $H \circ L$, and $L$ contains no critical events in $H \circ L$.

The next lemma states that if $n$ active processes are competing for entry into their critical sections, then at least $n-1$ of them execute at least one more critical event before entering their critical sections.

Lemma 5 Let $H$ be a computation. Assume the following:

- $H \in C$, and

- $H$ is regular (i.e., $\operatorname{Fin}(H)$ is a valid RF-set of $H$ ).

Define $n=|\operatorname{Act}(H)|$. Then, there exists a subset $Y$ of $\operatorname{Act}(H)$, where $n-1 \leq|Y| \leq n$, satisfying the following: for each process $p$ in $Y$, there exist a $p$-computation $L_{p}$ and an event $e_{p}$ by $p$ such that

- $H \circ L_{p} \circ\left\langle e_{p}\right\rangle \in C$;

- $L_{p}$ contains no critical events in $H \circ L_{p}$;

- $e_{p} \notin\left\{\right.$ Enter $_{p}, C S_{p}$, Exit $\left._{p}\right\}$;

- $\operatorname{Fin}(H)$ is a valid RF-set of $H \circ L_{p}$;

- $e_{p}$ is a critical event by $p$ in $H \circ L_{p} \circ\left\langle e_{p}\right\rangle$.

The following lemma is used to roll processes forward. It states that as long as there exist promoted processes, we can extend the computation with one more critical event of some promoted process, and at most one invisible process must be erased due to the resulting information flow.

Lemma 6 Consider a computation $H$ and set of processes RFS. Assume the following:

- $H \in C$;

- RFS is a valid RF-set of $H$;

- $\operatorname{Fin}(H) \subsetneq R F S$ (i.e., $\operatorname{Fin}(H)$ is a proper subset of RFS).

Then, there exists a computation $G$ satisfying the following.

- $G \in C$;

- RFS is a valid RF-set of $G$;

- $G$ can be written as $H \mid(Y \cup R F S) \circ L \circ\left\langle e_{p}\right\rangle$, for some choice of $Y, L$, and $e_{p}$, satisfying the following:

- $Y$ is a subset of $\operatorname{Inv}(H)$ such that $|\operatorname{Inv}(H)|-1 \leq|Y| \leq|\operatorname{Inv}(H)|$,

$-\operatorname{Inv}(G)=Y$,

- $L$ is a $\operatorname{Pmt}(H)$-computation,

- L has no critical events in $G$,

$-p \in \operatorname{Pmt}(H)$, and 
- $e_{p}$ is critical in $G$;

- $\operatorname{Pmt}(G) \subseteq \operatorname{Pmt}(H)$;

- An event in $H \mid(Y \cup R F S)$ is critical if and only if it is also critical in $H$.

The following theorem is due to Turán [38].

Theorem 1 (Turán) Let $\mathcal{G}=(V, E)$ be an undirected graph, with vertex set $V$ and edge set $E$. If the average degree of $\mathcal{G}$ is $d$, then an independent set ${ }^{16}$ exists with at least $\lceil|V| /(d+1)\rceil$ vertices.

The remaining lemma is unique to the lower bound established here and thus is presented with a full proof. This lemma provides the induction step that leads to the lower bound in Theorem 2.

Lemma 7 Let $k$ be a positive integer, and $H$ be a computation. Assume the following:

- $H \in C$, and

- $H$ is regular (i.e., $\operatorname{Fin}(H)$ is a valid RF-set of $H$ ).

Define $n=|\operatorname{Act}(H)|$. Also assume that

- $n>1$, and

- each process in $\operatorname{Act}(H)$ executes exactly c critical events in $H$.

Then, one of the following propositions is true.

Pr1: There exist a process $p$ in $\operatorname{Act}(H)$ and a computation $F$ in $C$ such that

- $F \circ\left\langle\right.$ Exit $\left._{p}\right\rangle \in C$;

- $F$ does not contain $\left\langle E_{\text {Exit }}\right\rangle$;

- at most $|\mathrm{Fin}(H)+2|$ processes participate in $F$;

- $p$ executes at least $k$ critical events in $F$.

Pr2: There exists a regular computation $G$ in $C$ such that

- $\operatorname{Act}(G) \subseteq \operatorname{Act}(H)$;

- $|\operatorname{Fin}(G)| \leq|\operatorname{Fin}(H)+2|$

- $|\operatorname{Act}(G)| \geq \min (\sqrt{n} /(2 c+3), \sqrt{n}-2 k-3)$;

- each process in $\operatorname{Act}(G)$ executes exactly $(c+1)$ critical events in $G$.

Proof: We first apply Lemma 5. Assumptions (29) and (30) stated in Lemma 5 follow from (49) and (50), respectively. It follows that there exists a set of processes $Y$ such that

- $Y \subseteq \operatorname{Act}(H)$, and

- $n-1 \leq|Y| \leq n$,

and for each process $p \in Y$, there exist a computation $L_{p}$ and an event $e_{p}$ by $p$, such that

- $H \circ L_{p} \circ\left\langle e_{p}\right\rangle \in C$;

- $L_{p}$ is a $p$-computation;

- $L_{p}$ contains no critical events in $H \circ L_{p}$;

- $e_{p} \notin\left\{\right.$ Enter $_{p}, C S_{p}$, Exit $\left._{p}\right\}$

- $\operatorname{Fin}(H)$ is a valid RF-set of $H \circ L_{p}$;

\footnotetext{
${ }^{16}$ An independent set of a graph $\mathcal{G}=(V, E)$ is a subset $V^{\prime} \subseteq V$ such that no edge in $E$ is incident to two vertices in $V^{\prime}$.
} 
- $e_{p}$ is a critical event by $p$ in $H \circ L_{p} \circ\left\langle e_{p}\right\rangle$.

For each $p \in Y$, by (60), (61), and $p \in Y \subseteq \operatorname{Act}(H)$, we have

$$
\operatorname{Act}\left(H \circ L_{p}\right)=\operatorname{Act}(H) \quad \wedge \quad \operatorname{Fin}\left(H \circ L_{p}\right)=\operatorname{Fin}(H) .
$$

By (51) and (58), $Y$ is nonempty.

If Proposition Pr1 is satisfied by any process in $Y$, then the theorem is clearly true. Thus, we will assume, throughout the remainder of the proof, that there is no process in $Y$ that satisfies $\operatorname{Pr} 1$. Define $\mathcal{E}_{H}$ as the set of critical events in $H$ of processes in $Y$.

$$
\mathcal{E}_{H}=\left\{f_{q} \text { in } H \mid f_{q} \text { is critical in } H \text { and } q \in Y\right\} .
$$

Define $\mathcal{E}=\mathcal{E}_{H} \cup\left\{e_{p} \mid p \in Y\right\}$, i.e., the set of all "past" and "next" critical events of processes in $Y$. From (52), (57), and (58), it follows that

$$
|\mathcal{E}|=(c+1)|Y| \leq(c+1) n .
$$

Define $V_{\text {next }}$ as the set of variables remotely accessed by some "next" critical events:

$$
V_{\text {next }}=\left\{v \in V \mid \text { there exists } p \in Y \text { such that } e_{p} \text { remotely accesses } v\right\} .
$$

We consider two cases, depending on the size of $V_{\text {next }}$.

\section{Case 1: $\left|V_{\text {next }}\right| \geq \sqrt{n}$ (erasing strategy)}

- In this case, we construct a subset $Y^{\prime}$ of $Y$ by selecting one process for each variable in $V_{\text {next }}$. Clearly, $\left|Y^{\prime}\right|=\left|V_{\text {next }}\right|$. We then construct a "conflict graph" $\mathcal{G}$, where each vertex is a process in $Y^{\prime}$. By applying Theorem 1, we can find a subset $Z$ of $Y^{\prime}$ such that their critical events do not conflict with each other. By applying Lemma 1 to $H$ and $Z \cup \operatorname{Fin}(H)$, and extending the resulting computation $H^{\prime}$ with next critical events, we construct a computation $G$ that satisfies Proposition Pr2.

By definition, for each variable $v$ in $V_{\text {next }}$, there exists a process $p$ in $Y$ such that $e_{p}$ remotely accesses $v$. Therefore, we can arbitrarily select one such process for each variable $v$ in $V_{\text {next }}$ and construct a set $Y^{\prime}$ of processes such that

- $Y^{\prime} \subseteq Y$,

- if $p \in Y^{\prime}, q \in Y^{\prime}$ and $p \neq q$, then $e_{p}$ and $e_{q}$ access different remote variables, and

- $\left|Y^{\prime}\right|=\left|V_{\text {next }}\right| \geq \sqrt{n}$.

We now construct an undirected graph $\mathcal{G}=\left(Y^{\prime}, E_{\mathcal{G}}\right)$, where each vertex is a process in $Y^{\prime}$. To each process $y$ in $Y^{\prime}$ and each variable $v \in \operatorname{var}\left(e_{y}\right)$ that is remote to $y$, we apply the following rules.

- R1: If $v$ is local to a process $z$ in $Y^{\prime}$, then introduce edge $\{y, z\}$.

- R2: If there exists an event $f_{p} \in \mathcal{E}$ that remotely accesses $v$, and if $p \in Y^{\prime}$, then introduce edge $\{y, p\}$.

Because each variable is local to at most one process, and since (by the Atomicity property) an event can access at most one remote variable, Rule R1 can introduce at most one edge per process. Since, by (52), y executes exactly $c$ critical events in $H$, by (70), Rule R2 can introduce at most $c$ edges per process.

Combining Rules R1 and R2, at most $c+1$ edges are introduced per process. Since each edge is counted twice (for each of its endpoints), the average degree of $\mathcal{G}$ is at most $2(c+1)$. Hence, by Theorem 1, there exists an independent set $Z$ such that

$$
Z \subseteq Y^{\prime}, \quad \text { and }
$$




$$
|Z| \geq\left|Y^{\prime}\right| /(2 c+3) \geq \sqrt{n} /(2 c+3),
$$

where the latter inequality follows from (71).

Next, we construct a computation $G$, satisfying Proposition $\operatorname{Pr} 2$, such that $\operatorname{Act}(G)=|Z|$.

Define $H^{\prime}$ as

$$
H^{\prime}=H \mid(Z \cup \operatorname{Fin}(H)) .
$$

By (57), (69), and (72), we have

$$
Z \subseteq Y^{\prime} \subseteq Y \subseteq \operatorname{Act}(H)
$$

and hence,

$$
\operatorname{Act}\left(H^{\prime}\right)=Z \subseteq \operatorname{Act}(H) \quad \wedge \quad \operatorname{Fin}\left(H^{\prime}\right)=\operatorname{Fin}(H) .
$$

We now apply Lemma 1, with ' $R F S^{\prime}$ ' $\leftarrow \operatorname{Fin}(H)$ and ' $Y$ ' $\leftarrow Z \cup \operatorname{Fin}(H)$. Among the assumptions stated in Lemma 1, (2) and (3) follow from (49) and (50), respectively; (4) is trivial. It follows that

- $H^{\prime} \in C$,

- $\operatorname{Fin}(H)$ is a valid RF-set of $H^{\prime}$, and

- an event in $H^{\prime}$ is critical if and only if it is also critical in $H$.

Our goal now is to show that $H^{\prime}$ can be extended so that each process in $Z$ has one more critical event. By (76), (78), and by the definition of a finished process,

$$
\operatorname{Inv}_{\operatorname{Fin}(H)}\left(H^{\prime}\right)=\operatorname{Act}\left(H^{\prime}\right)=Z .
$$

For each $z \in Z$, define $F_{z}$ as

$$
F_{z}=\left(H \circ L_{z}\right) \mid(Z \cup \operatorname{Fin}(H)) .
$$

By (75), we have $z \in Y$. Thus, applying (59), (60), (61), and (63) with ' $p$ ' $\leftarrow z$, it follows that

- $H \circ L_{z} \circ\left\langle e_{z}\right\rangle \in C$;

- $L_{z}$ is a $z$-computation;

- $L_{z}$ contains no critical events in $H \circ L_{z}$;

- $\operatorname{Fin}(H)$ is a valid RF-set of $H \circ L_{z}$.

By P1 (given in Section 2.1), (82) implies

$$
H \circ L_{z} \in C \text {. }
$$

We now apply Lemma 1, with ' $H$ ' $\leftarrow H \circ L_{z},{ }^{\prime} R F S$ ' $\leftarrow \operatorname{Fin}(H)$, and ' $Y$ ' $\leftarrow Z \cup \operatorname{Fin}(H)$. Among the assumptions stated in Lemma 1, (2) and (3) follow from (86) and (85), respectively; (4) is trivial. It follows that

- $F_{z} \in C$, and

- an event in $F_{z}$ is critical if and only if it is also critical in $H \circ L_{z}$.

Since $z \in Z$, by (74), (81), and (83), we have

$$
F_{z}=H^{\prime} \circ L_{z} .
$$

Hence, by (84) and (88),

- $L_{z}$ contains no critical events in $F_{z}=H^{\prime} \circ L_{z}$. 
Let $m=|Z|$ and index the processes in $Z$ as $Z=\left\{z_{1}, z_{2}, \ldots, z_{m}\right\}$. Define $L=L_{z_{1}} \circ L_{z_{2}} \circ \ldots \circ L_{z_{m}}$. We now use Lemma 4 , with ' $H$ ' $\leftarrow H^{\prime},{ }^{\prime} R F S^{\prime} \leftarrow \operatorname{Fin}(H),{ }^{\prime} Y$ ' $\leftarrow Z$, and ' $p_{j}$ ' $\leftarrow z_{j}$ for each $j=1, \ldots, m$. Among the assumptions stated in Lemma 4, (23)-(25) follow from (77), (78), and (80), respectively; (26)-(28) follow from (83), (87), and (89), respectively, with ' $z$ ' $\leftarrow z_{j}$ for each $j=1, \ldots, m$. This gives us the following.

- $H^{\prime} \circ L \in C$;

- $\operatorname{Fin}(H)$ is a valid RF-set of $H^{\prime} \circ L$;

- $L$ contains no critical events in $H^{\prime} \circ L$.

To this point, we have successfully appended a (possibly empty) sequence of noncritical events for each process in $Z$. It remains to append a "next" critical event for each such process. Note that, by (83) and the definition of $L$,

- $L$ is a $Z$-computation.

Thus, by (76) and (92), we have

$$
\operatorname{Act}\left(H^{\prime} \circ L\right)=\operatorname{Act}\left(H^{\prime}\right)=Z \quad \wedge \quad \operatorname{Fin}\left(H^{\prime} \circ L\right)=\operatorname{Fin}\left(H^{\prime}\right)=\operatorname{Fin}(H) .
$$

By (74) and the definition of $L$, it follows that

- for each $z \in Z,\left(H \circ L_{z}\right)\left|(\{z\} \cup \operatorname{Fin}(H))=\left(H^{\prime} \circ L\right)\right|(\{z\} \cup \operatorname{Fin}(H))$.

In particular, we have $\left(H \circ L_{z}\right)\left|z=\left(H^{\prime} \circ L\right)\right| z$. Therefore, by (82), (90), and repeatedly applying P3, it follows that, for each $z_{j} \in Z$, there exists an event $e_{z_{j}}^{\prime}$, such that

- $G \in C$, where $G=H^{\prime} \circ L \circ E$ and $E=\left\langle e_{z_{1}}^{\prime}, e_{z_{2}}^{\prime}, \ldots, e_{z_{m}}^{\prime}\right\rangle$;

- $e_{z_{j}}^{\prime} \sim e_{z_{j}}$.

By the definition of $E$,

- $E$ is a $Z$-computation.

By (62), (94), and (97), we have

$$
\operatorname{Act}(G)=\operatorname{Act}\left(H^{\prime} \circ L\right)=Z \quad \wedge \quad \operatorname{Fin}(G)=\operatorname{Fin}\left(H^{\prime} \circ L\right)=\operatorname{Fin}(H) .
$$

By (62), (64), and (97), it follows that for each $z_{j} \in Z$, both $e_{z_{j}}$ and $e_{z_{j}}^{\prime}$ access a common remote variable, say, $v_{j}$. Since $Z$ is an independent set of $\mathcal{G}$, by Rules $\mathrm{R} 1$ and $\mathrm{R} 2$, we have the following:

- for each $z_{j} \in Z, v_{j}$ is not local to any process in $Z$;

- $v_{j} \neq v_{k}$, if $j \neq k$.

Combining these two facts, we also have:

- for each $z_{j} \in Z$, no event in $E$ other than $e_{z_{j}}^{\prime}$ accesses $v_{j}$ (either locally or remotely).

We now establish two claims.

Claim 1: For each $z_{j} \in Z$, if we let $q=$ writer $\left(v_{j}, H^{\prime} \circ L\right)$, then one of the following holds: $q=\perp$, $q=z_{j}$, or $q \in \operatorname{Fin}(H)$.

Proof of Claim: It suffices to consider the case when $q \neq \perp$ and $q \neq z_{j}$ hold, in which case there exists an event $f_{q}$ by $q$ in $H^{\prime} \circ L$ that writes to $v_{j}$. By (74) and (93), we have $q \in Z \cup \operatorname{Fin}(H)$. We claim that $q \in \operatorname{Fin}(H)$ holds in this case. Assume, to the contrary,

$$
q \in Z .
$$


We consider two cases. First, if $f_{q}$ is a critical event in $H^{\prime} \circ L$, then by $(92), f_{q}$ is an event of $H^{\prime}$, and hence, by (79), $f_{q}$ is also a critical event in $H$. By (75) and (102), we have $q \in Y$. Thus, by (66), we have $f_{q} \in \mathcal{E}_{H}$, and hence $f_{q} \in \mathcal{E}$ holds by definition. By (100) and (102), $v_{j}$ is remote to $q$. Thus, $f_{q}$ remotely writes $v_{j}$. By (102) and $z_{j} \in Z$, we have

$$
\left\{q, z_{j}\right\} \subseteq Z,
$$

which implies $\left\{q, z_{j}\right\} \subseteq Y^{\prime}$ by (72). From this, our assumption of $q \neq z_{j}$, and by applying Rule R2 with ' $y$ ' $\leftarrow z_{j}$ and ' $f_{p}$ ' $\leftarrow f_{q}$, it follows that edge $\left\{q, z_{j}\right\}$ exists in $\mathcal{G}$. However, (103) then implies that $Z$ is not an independent set of $\mathcal{G}$, a contradiction.

Second, assume that $f_{q}$ is a noncritical event in $H^{\prime} \circ L$. Note that, by (100) and (102), $v_{j}$ is remote to $q$. Hence, by the definition of a critical event, there exists a critical event $\bar{f}_{q}$ by $q$ in $H^{\prime} \circ L$ that remotely writes to $v_{j}$. However, this leads to contradiction as shown above.

Claim 2: Every event in $E$ is critical in $G$. Also, $G$ satisfies RF5 with ' $R F S$ ' $\leftarrow \operatorname{Fin}(H)$.

Proof of Claim: Define $E_{0}=\langle\rangle$; for each positive $j$, define $E_{j}$ to be $\left\langle e_{z_{1}}^{\prime}, e_{z_{2}}^{\prime}, \ldots, e_{z_{j}}^{\prime}\right\rangle$, a prefix of $E$. We prove the claim by induction on $j$, applying Lemma 2 at each step. Note that, by (96) and P1, we have the following:

$$
H^{\prime} \circ L \circ E_{j} \circ\left\langle e_{z_{j+1}}^{\prime}\right\rangle=H^{\prime} \circ L \circ E_{j+1} \in C, \quad \text { for each } j .
$$

Also, by the definition of $E_{j}$, we have

$$
E_{j} \mid z_{j+1}=\langle\rangle, \quad \text { for each } j .
$$

At each step, we assume

- $H^{\prime} \circ L \circ E_{j}$ satisfies RF5 with 'RFS' $\leftarrow \operatorname{Fin}(H)$.

The induction base $(j=0)$ follows easily from (91), since $E_{0}=\langle\rangle$.

Assume that (106) holds for a particular value of $j$. Since $z_{j+1} \in Z$, by (75), we have

$$
z_{j+1} \in Y,
$$

and $z_{j+1} \in \operatorname{Act}(H)$. By applying (65) with ' $p$ ' $\leftarrow z_{j+1}$, and using (107), we also have $\operatorname{Act}\left(H \circ L_{z_{j+1}}\right)=$ $\operatorname{Act}(H)$, and hence

$$
z_{j+1} \in \operatorname{Act}\left(H \circ L_{z_{j+1}}\right) .
$$

By (105), if any event $e_{z_{k}}^{\prime}$ in $E_{j}$ accesses a local variable $v$ of $z_{j+1}$, then $e_{z_{k}}^{\prime}$ accesses $v$ remotely, and hence $v=v_{k}$ by definition. However, by (100), $v_{k}$ cannot be local to $z_{j+1}$. It follows that

- no events in $E_{j}$ access any of $z_{j+1}$ 's local variables.

We now apply Lemma 2 , with ' $H$ ' $\leftarrow H \circ L_{z_{j+1}}$, ' $H^{\prime}$ ' $\leftarrow H^{\prime} \circ L$, 'G' $\leftarrow E_{j}$, 'RFS' $\leftarrow \operatorname{Fin}(H)$, ' $e_{p}$ ' $\leftarrow$ $e_{z_{j+1}}$, and ' $e_{p}^{\prime}$ ' $\leftarrow e_{z_{j+1}}^{\prime}$. Among the assumptions stated in Lemma 2, (6), (8), (10), (12), and (13) follow from (104), (91), (108), (105), and (109), respectively; (9) follows by applying (97) with ' $z_{j}$ ' $\leftarrow z_{j+1}$; (7) and (11) follow by applying (85) and (95), respectively, with ' $z$ ' $\leftarrow z_{j+1}$; and (5) and (14) follow by applying (59) and (64), respectively, with ' $p$ ' $\leftarrow z_{j+1}$, and using (107). Moreover, 
Assumption (A) follows from (106), and Assumption (B) is satisfied vacuously (with ' $v$ ' $\leftarrow v_{j+1}$ ) by (101).

It follows that $e_{z_{j+1}}^{\prime}$ is critical in $H^{\prime} \circ L \circ E_{j} \circ\left\langle e_{z_{j+1}}^{\prime}\right\rangle=H^{\prime} \circ L \circ E_{j+1}$, and that $H^{\prime} \circ L \circ E_{j+1}$ satisfies RF5 with 'RFS' $\leftarrow \operatorname{Fin}(H)$.

We now claim that $\operatorname{Fin}(H)$ is a valid RF-set of $G$. Condition RF5 was already proved in Claim 2.

- RF1 and RF2: Define $E_{j}$ as in Claim 2. We establish RF1 and RF2 by induction on $j$, applying Lemma 3 at each step. At each step, we assume

- $H^{\prime} \circ L \circ E_{j}$ satisfies RF1 and RF2 with 'RFS' $\leftarrow \operatorname{Fin}(H)$.

The induction base $(j=0)$ follows easily from $(91)$, since $E_{0}=\langle\rangle$.

Assume that (110) holds for a particular value of $j$. Note that, by (101), we have $\operatorname{writer}\left(v_{j+1}, H^{\prime} \circ L \circ E_{j}\right)=$ writer $\left(v_{j+1}, H^{\prime} \circ L\right)$. Thus, by (94) and Claim 1 ,

- if we let $q=\operatorname{writer}\left(v_{j+1}, H^{\prime} \circ L \circ E_{j}\right)$, then one of the following holds: $q=\perp, q=z_{j+1}$, or $q \in \operatorname{Fin}(H)=$ $\operatorname{Fin}\left(H^{\prime} \circ L\right)$.

We now apply Lemma 3 , with ' $H$ ' $\leftarrow H^{\prime} \circ L$, ' $G$ ' $\leftarrow E_{j},{ }^{\prime} R F S$ ' $\leftarrow \operatorname{Fin}(H),{ }^{\prime} e_{p}$ ' $\leftarrow e_{z_{j+1}}^{\prime}$, and ' $v_{\text {rem }}$ ' $\leftarrow v_{j+1}$. Among the assumptions stated in Lemma 3, (15), (16), (18), (20), and (22) follow from (104), (91), (110), (105), and (111), respectively; (17) follows from (94) and $z_{j+1} \in Z$; (19) follows from (94) and (98); (21) follows from (100) and (94). It follows that $H^{\prime} \circ L \circ E_{j+1}$ satisfies RF1 and RF2 with ' $R F S$ ' $\leftarrow$ Fin $(H)$.

- RF3: Consider a variable $v \in V$ and two different events $f_{q}$ and $g_{r}$ in $G$. Assume that both $q$ and $r$ are in $\operatorname{Act}(G), q \neq r$, and that there exists a variable $v$ such that $v \in \operatorname{var}\left(f_{q}\right) \cap \operatorname{var}\left(g_{r}\right)$. (Note that, by (99), $\{q, r\} \subseteq Z$.) We claim that these conditions can actually never arise simultaneously, which implies that $G$ vacuously satisfies RF3.

Since $v$ is remote to at least one of $q$ or $r$, without loss of generality, assume that $v$ is remote to $q$. We claim that there exists an event $\bar{f}_{q}$ in $\mathcal{E}$ that accesses the same variable $v$. If $f_{q}$ is an event of $E$, we have $f_{q}=e_{z_{j}}^{\prime}$ for some $z_{j} \in Z$, and $e_{z_{j}} \in \mathcal{E}$ holds by definition; define $\bar{f}_{q}=e_{z_{j}}$ in this case. If $f_{q}$ is a noncritical event in $H^{\prime} \circ L$, then by the definition of a critical event, there exists a critical event $\bar{f}_{q}$ in $H^{\prime} \circ L$ that remotely accesses $v$. If $f_{q}$ is a critical event in $H^{\prime} \circ L$, then define $\bar{f}_{q}=f_{q}$. (Note that, if $\bar{f}_{q}$ is a critical event in $H^{\prime} \circ L$, then by (79) and (92), $\bar{f}_{q}$ is also a critical event in $H$, and hence, by $q \in Z$, (75), and the definition of $\mathcal{E}$, we have $\bar{f}_{q} \in \mathcal{E}$.)

It follows that, in each case, there exists an event $\bar{f}_{q} \in \mathcal{E}$ that remotely accesses $v$. If $v$ is local to $r$, then by Rule R1, $\mathcal{G}$ contains the edge $\{q, r\}$. On the other hand, if $v$ is remote to $r$, then we can choose an event $\bar{g}_{r} \in \mathcal{E}$ that remotely accesses $v$, in the same way as shown above. Hence, by Rule R2, $\mathcal{G}$ contains the edge $\{q, r\}$. Thus, in either case, $p$ and $q$ cannot simultaneously belong to $Z$, a contradiction.

- RF4: By (91) and (99), it easily follows that $G$ satisfies RF4 with respect to Fin $(H)$.

Finally, we claim that $G$ satisfies Proposition Pr2. By (99), which implies $\operatorname{Act}(G)=Z \subseteq \operatorname{Act}(H), G$ satisfies (53) and (54). By (73), we have (55). By (52), (79), and (92), each process in $Z$ executes exactly $c$ critical events in $H^{\prime} \circ L$. Thus, by Claim 2, G satisfies (56).

\section{Case 2: $\left|V_{\text {next }}\right| \leq \sqrt{n}$ (roll-forward strategy)}

- In this case, there exists a variable $v$ that is remotely accessed by next critical events of at least $\sqrt{n}-1$ processes. Let 
$Y_{v}$ be the set of these processes. We retain $Y_{v}$ and erase all other active processes. Let the resulting computation be $H^{\prime}$. We then roll forward processes $p_{\mathrm{LW}}$ and $p_{\mathrm{SC}}$ of $Y_{v}$ to generate a regular computation $G$. If either $p_{\mathrm{LW}}$ or $p_{\mathrm{SC}}$ executes $k$ or more critical events before finishing its execution, the resulting computation satisfies Proposition Pr1. Otherwise, fewer than $2 k$ processes are erased during the procedure, which makes $G$ satisfy Proposition Pr 2 , with at least $\sqrt{n}-2 k$ active processes.

For each variable $v_{j}$ in $V_{\text {next }}$, define $Y_{v_{j}}=\left\{p \in Y \mid e_{p}\right.$ remotely accesses $\left.v_{j}\right\}$. By (58) and $(68),\left|V_{\text {next }}\right| \leq \sqrt{n}$ implies that there exists a variable $v$ in $V_{\text {next }}$ such that $\left|Y_{v}\right| \geq(n-1) / \sqrt{n}$ holds. (In the rest of Case 2 , we consider $v$ to be a fixed variable.) Then, the following holds:

$$
\left|Y_{v}\right| \geq(n-1) / \sqrt{n}>\sqrt{n}-1
$$

Define

$$
H^{\prime}=H \mid\left(Y_{v} \cup \operatorname{Fin}(H)\right) \text {. }
$$

Using $Y_{v} \subseteq Y \subseteq \operatorname{Act}(H)$, we also have

$$
\operatorname{Act}\left(H^{\prime}\right)=Y_{v} \subseteq \operatorname{Act}(H) \wedge \operatorname{Fin}\left(H^{\prime}\right)=\operatorname{Fin}(H)
$$

We now apply Lemma 1 , with ' $R F S$ ' $\leftarrow \operatorname{Fin}(H)$ and ' $Y$ ' $\leftarrow Y_{v} \cup \operatorname{Fin}(H)$. Among the assumptions stated in Lemma 1, (2) and (3) follow from (49) and (50), respectively; (4) is trivial. It follows that

- $H^{\prime} \in C$,

- $\operatorname{Fin}(H)$ is a valid RF-set of $H^{\prime}$, and

- an event in $H^{\prime}$ is critical if and only if it is also critical in $H$.

Our goal now is to show that $H^{\prime}$ can be extended to a computation $\bar{G}$ (defined later), so that each process in $Y_{v}$ has one more critical event. By (114), (116), and by the definition of a finished process,

$$
\operatorname{Inv}_{\operatorname{Fin}(H)}\left(H^{\prime}\right)=\operatorname{Act}\left(H^{\prime}\right)=Y_{v}
$$

For each $s \in Y_{v}$, define $F_{s}$ as

$$
F_{s}=\left(H \circ L_{s}\right) \mid\left(Y_{v} \cup \operatorname{Fin}(H)\right) .
$$

Since $Y_{v} \subseteq Y$, we have $s \in Y$. Thus, applying (59), (60), (61), and (63) with ' $p$ ' $\leftarrow s$, it follows that

- $H \circ L_{s} \circ\left\langle e_{s}\right\rangle \in C$;

- $L_{s}$ is an $s$-computation;

- $L_{s}$ contains no critical events in $H \circ L_{s}$;

- $\operatorname{Fin}(H)$ is a valid RF-set of $H \circ L_{s}$.

By P1, (120) implies

$$
H \circ L_{s} \in C \text {. }
$$

We now apply Lemma 1 , with ' $H$ ' $\leftarrow H \circ L_{s}, ' R F S$ ' $\leftarrow \operatorname{Fin}(H)$, and ' $Y$ ' $\leftarrow Y_{v} \cup \operatorname{Fin}(H)$. Among the assumptions stated in Lemma 1, (2) and (3) follow from (124) and (123), respectively; (4) is trivial. It follows that

- $F_{s} \in C$, and

- an event in $F_{s}$ is critical if and only if it is also critical in $H \circ L_{s}$.

Since $s \in Y_{v}$, by (113), (119), (121), and (125), we have 
- $F_{s}=H^{\prime} \circ L_{s} \in C$.

Hence, by (122) and (126),

- $L_{s}$ contains no critical events in $F_{s}=H^{\prime} \circ L_{s}$.

We now show that the events in $\left\{L_{s} \mid s \in Y_{v}\right\}$ can be "merged" by applying Lemma 4. We arbitrarily index $Y_{v}$ as $\left\{s_{1}, s_{2}, \ldots, s_{m}\right\}$, where $m=\left|Y_{v}\right|$. (Later, we construct a specific indexing of $Y_{v}$ to reduce information flow.) Let $L=L_{s_{1}} \circ L_{s_{2}} \circ \cdots \circ L_{s_{m}}$. Apply Lemma 4, with ' $H$ ' $\leftarrow H^{\prime}$, ' $R F S$ ' $\leftarrow$ Fin $(H)$, ' $Y$ ' $\leftarrow Y_{v}$, and ' $p_{j}$ ' $\leftarrow s_{j}$ for each $j=1, \ldots, m$. Among the assumptions stated in Lemma 4, (23)-(25) follow from (115), (116), and (118), respectively; (26)-(28) follow from (121), (127), and (128), respectively, with ' $s$ ' $\leftarrow s_{j}$ for each $j=1, \ldots, m$. This gives us the following.

- $H^{\prime} \circ L \in C$;

- $\operatorname{Fin}(H)$ is a valid RF-set of $H^{\prime} \circ L$;

- $L$ contains no critical events in $H^{\prime} \circ L$.

By (113) and the definition of $L$, we also have,

- for each $s \in Y_{v},\left(H \circ L_{s}\right)\left|(\{s\} \cup \operatorname{Fin}(H))=\left(H^{\prime} \circ L\right)\right|(\{s\} \cup \operatorname{Fin}(H))$;

- for each $s \in Y_{v},\left(H^{\prime} \circ L\right)\left|s=\left(H \circ L_{s}\right)\right| s$.

We now re-index the processes in $Y_{v}$ so that information flow among them is minimized. We place next critical events of $Y_{v}$ by placing write, comparison, and read events in that order. Furthermore, we can arrange comparison events such that at most one of them succeeds, as explained in Section 3. Let $\left(s^{1}, s^{2}, \ldots, s^{m}\right)$ be the indexing of $Y_{v}$ thus constructed, and $E$ be the appended computation that consists of next critical events by processes in $Y$. Then, we have the following:

- $\bar{G} \in C$, where $\bar{G}=H^{\prime} \circ L \circ E$ and $E=\left\langle e_{s^{1}}^{\prime}, e_{s^{2}}^{\prime}, \ldots, e_{s^{m}}^{\prime}\right\rangle$;

- $e_{s^{j}}^{\prime} \sim e_{s^{j}}$.

By the definition of $E$,

- $E$ is an $Y_{v}$-computation.

By (62), (131), and (135), $L \circ E$ does not contain any transition events. Moreover, by the definition of $L$ and $E,(L \circ E) \mid p \neq\langle\rangle$ implies $p \in Y_{v}$, for each process $p$. Combining these assertions with (114), we have

$$
\begin{aligned}
& \operatorname{Act}(\bar{G})=\operatorname{Act}\left(H^{\prime} \circ L\right)=\operatorname{Act}\left(H^{\prime}\right)=Y_{v} \wedge \\
& \operatorname{Fin}(\bar{G})=\operatorname{Fin}\left(H^{\prime} \circ L\right)=\operatorname{Fin}\left(H^{\prime}\right)=\operatorname{Fin}(H) .
\end{aligned}
$$

We now state and prove two claims regarding $\bar{G}$. Claim 3 follows easily from the re-indexing of $Y_{v}$ and construction of $E$, described above.

Claim 3: Events in $E$ appear in the following order, where $\alpha$ is a fixed value in the range of $v$ and $W(v), C_{1}(v), C_{2}(v)$, and $R(v)$ are sets of events.

- events in $W(v)$ : each event $e_{s}^{\prime}$ in $W(v)$ satisfies $o p\left(e_{s}^{\prime}\right)=$ write $(v)$;

- events in $C_{1}(v)$ : each event $e_{s}^{\prime}$ in $C_{1}(v)$ satisfies op $\left(e_{s}^{\prime}\right)=\operatorname{compare}\left(v, \beta_{s}\right)$ for some $\beta_{s} \neq \alpha$;

- events in $C_{2}(v)$ : each event $e_{s}^{\prime}$ in $C_{2}(v)$ satisfies op $\left(e_{s}^{\prime}\right)=\operatorname{compare}(v, \alpha)$;

- events in $R(v)$ : each event $e_{s}^{\prime}$ in $R(v)$ satisfies $o p\left(e_{s}^{\prime}\right)=\operatorname{read}(v)$.

Moreover, in the computation $\bar{G}$, after all events in $W(v)$ are executed, and before any event in $C_{2}(v)$ is executed, $v$ has the value $\alpha$. All events in $C_{1}(v)$ (if any) are unsuccessful comparisons. At most one 
event in $C_{2}(v)$ is a successful comparison. (Note that a successful comparison event writes a value other than $\alpha$, by definition. Thus, if there is a successful comparison, then all subsequent comparison events must fail.) Define $L W(v)$, the "last write," and $S C(v)$, the "successful comparison," as follows:

$$
\begin{aligned}
& L W(v)= \begin{cases}\text { the last event in } W(v), & \text { if } W(v) \neq\{\}, \\
\text { writer_event }\left(v, H^{\prime} \circ L\right), & \text { if } W(v)=\{\} ;\end{cases} \\
& S C(v)= \begin{cases}\text { the successful comparison in } C_{2}(v), & \text { if } C_{2}(v) \text { contains one, } \\
\perp, & \text { otherwise. }\end{cases}
\end{aligned}
$$

Then, the last process to write to $v$ (if any) is either $S C(v)$ (if $S C(v)$ is defined) or $L W(v)$ (otherwise).

Before establishing our next claim, Claim 4, we define $p_{\mathrm{LW}}$ and $p_{\mathrm{SC}}$ as the processes that execute $L W(v)$ and $S C(v)$, respectively. If $L W(v)$ (respectively, $S C(v)$ ) equals $\perp$, then $p_{\mathrm{LW}}$ (respectively, $p_{\mathrm{SC}}$ ) also equals $\perp$. We also define $R F S$ as

$$
R F S=\operatorname{Fin}(H) \cup\left\{p \mid p \in\left\{p_{\mathrm{LW}}, p_{\mathrm{SC}}\right\} \text { and } p \neq \perp\right\} .
$$

By the definition of $Y_{v}$, for each $p \in Y_{v}, e_{p}$ remotely accesses $v$. In particular,

- for each $p \in Y_{v}, v$ is remote to $p$.

Note that "expanding" a valid RF-set does not falsify any of RF1-RF5. Therefore, using (130), (137), and $\operatorname{Fin}(H) \subseteq R F S \subseteq \operatorname{Fin}(H) \cup Y_{v}$, it follows that

- $R F S$ is a valid RF-set of $H^{\prime} \circ L$.

We now establish Claim 4, stated below.

Claim 4: Every event in $E$ is critical in $\bar{G}$. Also, $\bar{G}$ satisfies RF5.

Proof of Claim: Define $E_{0}=\langle\rangle$; for each positive $j$, define $E_{j}$ to be $\left\langle e_{s^{1}}^{\prime}, e_{s^{2}}^{\prime}, \ldots, e_{s^{j}}^{\prime}\right\rangle$, a prefix of $E$. We prove the claim by induction on $j$, applying Lemma 2 at each step. Note that, by (134) and P1, we have the following:

$$
H^{\prime} \circ L \circ E_{j} \circ\left\langle e_{s^{j+1}}^{\prime}\right\rangle=H^{\prime} \circ L \circ E_{j+1} \in C, \quad \text { for each } j .
$$

Also, by the definition of $E_{j}$, we have

$$
E_{j} \mid s^{j+1}=\langle\rangle, \quad \text { for each } j .
$$

At each step, we assume

- $H^{\prime} \circ L \circ E_{j}$ satisfies RF5.

The induction base $(j=0)$ follows easily from (140), since $E_{0}=\langle\rangle$.

Assume that (143) holds for a particular value of $j$. Since $s^{j+1} \in Y_{v} \subseteq Y$, we have

$$
s^{j+1} \in Y,
$$

and $s^{j+1} \in \operatorname{Act}(H)$. By applying (65) with ' $p$ ' $\leftarrow s^{j+1}$, and using (144), we also have $\operatorname{Act}\left(H \circ L_{s^{j+1}}\right)=$ $\operatorname{Act}(H)$, and hence

$$
s^{j+1} \in \operatorname{Act}\left(H \circ L_{s^{j+1}}\right) .
$$

Also, by (139), 
- no events in $E_{j}$ access any of $s^{j+1}$ 's local variables.

We use Lemma 2 twice in sequence in order to prove Claim 4. First, by P3, and applying (120), (129), and (133) with ' $s$ ' $\leftarrow s^{j+1}$, it follows that there exists an event $e_{s^{j+1}}^{\prime \prime}$, such that

- $H^{\prime} \circ L \circ\left\langle e_{s^{j+1}}^{\prime \prime}\right\rangle \in C$, and

- $e_{s^{j+1}}^{\prime \prime} \sim e_{s^{j+1}}$.

We now apply Lemma 2 , with ' $H$ ' $\leftarrow H \circ L_{s^{j+1}},{ }^{\prime} H^{\prime}$ ' $\leftarrow H^{\prime} \circ L$, 'G' $\leftarrow\langle\rangle,{ }^{\prime} R F S^{\prime} \leftarrow \operatorname{Fin}(H)$, ' $e_{p}$ ' $\leftarrow$ $e_{s^{j+1}}$, and ' $e_{p}^{\prime}$ ' $\leftarrow e_{s^{j+1}}^{\prime \prime}$. Among the assumptions stated in Lemma 2, (6) and (8)-(10) follow from (147), (130), (148), and (145), respectively; (12) and (13) hold vacuously by ' $G$ ' $\leftarrow\langle\rangle$; (5), (7), and (11) follow by applying (120), (123), and (132), respectively, with ' $s$ ' $\leftarrow s^{j+1}$; (14) follows by applying (64) with ' $p$ ' $\leftarrow s^{j+1}$, and using (144). It follows that

- $e_{s^{j+1}}^{\prime \prime}$ is critical in $H^{\prime} \circ L \circ\left\langle e_{s^{j+1}}^{\prime \prime}\right\rangle$.

Before applying Lemma 2 again, we establish the following preliminary assertions. Since $\operatorname{Fin}(H) \subseteq$ $R F S$, by applying (123) with ' $s$ ' $\leftarrow s^{j+1}$, it follows that

- RFS is a valid RF-set of $H \circ L_{s^{j+1}}$.

We now establish a simple claim.

Claim 4-1: If $e_{s^{j+1}}$ is a comparison event on $v$, and if $E_{j}$ contains a write to $v$, then $E_{j} \mid R F S$ also contains a write to $v$.

Proof of Claim: By (135) and Claim 3, we have $e_{s^{j+1}}^{\prime} \in C_{1}(v) \cup C_{2}(v)$. Hence, by Claim 3, if an event $e_{s^{k}}^{\prime}$ (for some $k \leq j$ ) in $E_{j}$ writes to $v$, then we have either $e_{s^{k}}^{\prime} \in W(v)$ or $e_{s^{k}}^{\prime}=S C(v)$. If $e_{s^{k}}^{\prime}=S C(v)$, then since $s^{k} \in R F S$ holds by (138), Claim 4-1 is satisfied. On the other hand, if $e_{s^{k}}^{\prime} \in W(v)$, then $W(v)$ is nonempty. Moreover, since all events in $W(v)$ are indexed before any events in $C_{1}(v) \cup C_{2}(v), E_{j}$ contains all events in $W(v)$. Thus, by (138), both $E_{j}$ and $E_{j} \mid R F S$ contain $L W(v)$, an event that writes to $v$.

We now apply Lemma 2 again, with ' $H$ ' $\leftarrow H^{\prime} \circ L$, ' $H^{\prime}$ ' $\leftarrow H^{\prime} \circ L,{ }^{\prime} G^{\prime} \leftarrow E_{j}$, ' $e_{p}$ ' $\leftarrow e_{s^{j+1}}^{\prime \prime}$, and ' $e_{p}^{\prime}$ ' $\leftarrow e_{s^{j+1}}^{\prime}$. Among the assumptions stated in Lemma 2, (5)-(8) and (12)-(14) follow from (147), (141), (140), (140), (142), (146), and (149), respectively; (11) is trivial; (9) follows from (148) and by applying (135) with ' $s^{j}$ ' $\leftarrow s^{j+1}$; (10) follows from (137) and $s^{j+1} \in Y_{v}$. Moreover, Assumption (A) follows from (143), and Assumption (B) follows from Claim 4-1.

It follows that $e_{s^{j+1}}^{\prime}$ is critical in $H^{\prime} \circ L \circ E_{j} \circ\left\langle e_{s^{j+1}}^{\prime}\right\rangle=H^{\prime} \circ L \circ E_{j+1}$, and that $H^{\prime} \circ L \circ E_{j+1}$ satisfies RF5.

We now show that $R F S$ is a valid RF-set of $\bar{G}$. Condition RF5 was already proved in Claim 4 .

- RF1 and RF2: Define $E_{j}$ as in Claim 4. We establish RF1 and RF2 by induction on $j$, applying Lemma 3 at each step. At each step, we assume

- $H^{\prime} \circ L \circ E_{j}$ satisfies RF1 and RF2.

The induction base $(j=0)$ follows easily from (140), since $E_{0}=\langle\rangle$.

Assume that (151) holds for a particular value of $j$. By Claim 3, if $e_{s^{j+1}}^{\prime}$ reads $v$, then the following holds: $e_{s^{j+1}}^{\prime} \in C_{1}(v) \cup C_{2}(v) \cup R(v)$; every event in $W(v)$ is contained in $E_{j}$; writer $\left(v, H^{\prime} \circ L \circ E_{j}\right)$ is one of $L W(v)$ or $S C(v)$ or $\perp$. Therefore, by (138), we have the following: 
- if $e_{s^{j+1}}^{\prime}$ remotely reads $v$, and if we let $q=\operatorname{writer}\left(v, H^{\prime} \circ L \circ E_{j}\right)$, then either $q=\perp$ or $q \in R F S$ holds.

We now apply Lemma 3, with ' $H$ ' $\leftarrow H^{\prime} \circ L, ~ ' G$ ' $\leftarrow E_{j}$, ' $e_{p}$ ' $\leftarrow e_{s^{j+1}}^{\prime}$, and ' $v_{\text {rem }}$ ' $\leftarrow v$. Among the assumptions stated in Lemma 3, (15), (16), (18), (20), and (22) follow from (141), (140), (151), (142), and (152), respectively; (17) follows from (137) and $s^{j+1} \in Y_{v}$; (19) follows from (137) and (136); (21) follows from (137) and (139). It follows that $H^{\prime} \circ L \circ E_{j+1}$ satisfies RF1 and RF2.

- RF3: Consider a variable $u \in V$ and two different events $f_{p}$ and $g_{q}$ in $\bar{G}$. Assume that both $p$ and $q$ are in $\operatorname{Act}(\bar{G}), p \neq q$, that there exists a variable $u$ such that $u \in \operatorname{var}\left(f_{p}\right) \cup \operatorname{var}\left(g_{q}\right)$, and that there exists a write to $u$ in $\bar{G}$. Define $r=\operatorname{writer}(u, \bar{G})$. Our proof obligation is to show that $r \in R F S$.

By (137), we have $\{p, q\} \subseteq Y_{v}$. If $u=v$, then by Claim 3, writer_event $(u, \bar{G})$ is either $S C(u)$ (if $\left.S C(u) \neq \perp\right)$ or $L W(u)$ (otherwise). (Since we assumed that there exists a write to $u$, they both cannot be $\perp$.) Thus, by (138), we have $r \in R F S$.

On the other hand, assume $u \neq v$. We now consider three cases.

- Consider the case in which both $f_{p}$ and $g_{q}$ are in $H^{\prime} \circ L$.

If there exists an event $e_{s}^{\prime}$ in $E$ such that $u \in \operatorname{Wvar}\left(e_{s}^{\prime}\right)$, then since $u \neq v, u$ is local to $s$. Since at least one of $p$ or $q$ is different from $s$, without loss of generality, assume $p \neq s$. Since $p \in Y_{v}$ and $Y_{v} \subseteq \operatorname{Act}(H)$, we have $p \notin \operatorname{Fin}(H)$. Thus, by (130) and by applying RF2 with ' $R F S$ ' $\leftarrow \operatorname{Fin}(H)$ to $f_{p}$ in $H^{\prime} \circ L$, we have $s \notin \operatorname{Act}\left(H^{\prime} \circ L\right)$. However, by (137), $\operatorname{Act}\left(H^{\prime} \circ L\right)=Y_{v}$, which contradicts $s \in Y_{v}$ (which follows from (136), since $e_{s}^{\prime}$ is an event of $E$ ).

It follows that there exists no event $e_{s}^{\prime}$ in $E$ such that $u \in \operatorname{Wvar}\left(e_{s}^{\prime}\right)$ holds. Thus, we have $r=$ writer $\left(u, H^{\prime} \circ L\right)$. By (130) and applying RF3 with 'RFS' $\leftarrow$ Fin $(H)$ to $f_{p}$ and $g_{q}$ in $H^{\prime} \circ L$, we have writer $\left(u, H^{\prime} \circ L\right) \in \operatorname{Fin}(H) \subseteq R F S$.

- Consider the case in which $f_{p}$ is in $H^{\prime} \circ L$ and $g_{q}=e_{s^{k}}^{\prime}$, for some $s^{k} \in Y_{v}$. By (137) and our assumption that $p$ and $q$ are both in $\operatorname{Act}(\bar{G})$, we have $p \in \operatorname{Act}\left(H^{\prime} \circ L\right)$ and $q \in \operatorname{Act}\left(H^{\prime} \circ L\right)$. Since $u \neq v, u$ is local to $q$. However, by (130), and by applying RF2 with ' $R F S$ ' $\leftarrow$ Fin $(H)$ to $f_{p}$ in $H^{\prime} \circ L$, we have $q \notin \operatorname{Act}\left(H^{\prime} \circ L\right)$, a contradiction.

- Consider the case in which $f_{p}=e_{s^{j}}^{\prime}$ and $g_{q}=e_{s^{k}}^{\prime}$, for some $s^{j}$ and $s^{k}$ in $Y_{v}$. Since $u$ is remote to at least one of $s^{j}$ or $s^{k}$, we have $u=v$, a contradiction.

- RF4: By (62), (130), and (137), it easily follows that $\bar{G}$ satisfies RF4 with respect to $R F S$.

Therefore, we have established that

- $R F S$ is a valid RF-set of $\bar{G}$.

By (137) and (138), we have

$$
\operatorname{Pmt}_{R F S}(\bar{G})=\left\{p \mid p \in\left\{p_{\mathrm{LW}}, p_{\mathrm{SC}}\right\} \text { and } p \neq \perp\right\} .
$$

In particular,

$$
\left|\operatorname{Pmt}_{R F S}(\bar{G})\right| \leq 2 .
$$

We now let the processes in $\operatorname{Pmt}(\bar{G})$ finish their execution by inductively appending critical events of processes in $\operatorname{Pmt}(\bar{G})$, thus generating a sequence of computations $G_{0}, G_{1}, \ldots, G_{l}$ (where $G_{0}=\bar{G}$ ), satisfying the following: 
- $G_{j} \in C$;

- $R F S$ is a valid RF-set of $G_{j}$;

- $\operatorname{Pmt}\left(G_{j}\right) \subseteq \operatorname{Pmt}(\bar{G})$;

- each process in $\operatorname{Inv}\left(G_{j}\right)$ executes exactly $c+1$ critical events in $G_{j}$;

- the processes in $\operatorname{Pmt}(\bar{G})$ collectively execute exactly $|\operatorname{Pmt}(\bar{G})| \cdot(c+1)+j$ critical events in $G_{j}$;

- $\operatorname{Inv}\left(G_{j+1}\right) \subseteq \operatorname{Inv}\left(G_{j}\right)$ and $\mid \operatorname{Inv}\left(G_{j+1}|\geq| \operatorname{Inv}\left(G_{j}\right) \mid-1\right.$ if $j<l$;

- $\operatorname{Fin}\left(G_{j}\right) \subsetneq R F S$ if $j<l$, and $\operatorname{Fin}\left(G_{j}\right)=R F S$ if $j=l$.

At each induction step, we apply Lemma 6 to $G_{j}$ in order to construct $G_{j+1}$, until $\operatorname{Fin}\left(G_{j}\right)=R F S$ is established, at which point the induction is completed. The induction is explained in detail below.

Induction base $(j=0)$ : Since $G_{0}=\bar{G},(155)$ and (156) follow from (134) and (153), respectively. Condition (157) is trivial.

By (52), (117), and (131), each process in $Y_{v}$ executes exactly $c$ critical events in $H^{\prime} \circ L$. Thus, by Claim 4 , it follows that each process in $Y_{v}$ executes exactly $c+1$ critical events in $\bar{G}$. Since $\operatorname{Inv}(\bar{G}) \subseteq Y_{v}, \bar{G}$ satisfies (158). Since $\operatorname{Pmt}(\bar{G}) \subseteq Y_{v}, \bar{G}$ satisfies (159).

Induction step: At each step, we assume (155)-(159). If Fin $\left(G_{j}\right)=R F S$, then (161) is satisfied and we finish the induction, by letting $l=j$.

Assume otherwise. We apply Lemma 6 with ' $H$ ' $\leftarrow G_{j}$. Assumptions (36)-(38) stated in Lemma 6 follow from (155), (156), and $\operatorname{Fin}\left(G_{j}\right) \neq R F S$. The lemma implies that a computation $G_{j+1}$ exists satisfying (155)-(161), as shown below.

Condition (155) and (156) follow from (39) and (40), respectively. Since $G_{j}$ satisfies (157), by (47), $G_{j+1}$ also satisfies (157). Since $\operatorname{Inv}\left(G_{j+1} \subseteq \operatorname{Inv}\left(G_{j}\right)\right.$ by (41) and (42), by (44) and (48), and applying (158) to $G_{j}$, it follows that $G_{j+1}$ satisfies (158). By (44)-(48), and applying (157) and (159) to $G_{j}$, it follows that $G_{j+1}$ satisfies (159). Condition (160) follows from (41) and (42). Thus, the induction is established.

We now show that $l<2 k$. Assume otherwise. By (154), and by applying (159) to $G_{l}$, it follows that there exists a process $p \in \operatorname{Pmt}(\bar{G})$ (i.e., $p$ is either $p_{\mathrm{LW}}$ or $p_{\mathrm{SC}}$ ) such that $p$ executes at least $c+1+k$ critical events in $G_{l}$. From $(161)$ and $p \in \operatorname{Pmt}(\bar{G}) \subseteq R F S$, we get $p \in \operatorname{Fin}\left(G_{l}\right)$. Let $\bar{F}=G_{l} \mid R F S$. By Lemma 1 , and applying (155) and (156), we have the following:

- $\bar{F} \in C$;

- $R F S$ is a valid RF-set of $\bar{F}$;

- $p$ executes at least $c+1+k$ critical events in $\bar{F}$.

Since $p \in \operatorname{Fin}\left(G_{l}\right)$, by applying RF4 to $p$ in $G_{l}$, it follows that the last event of $G_{l} \mid p$ is Exit ${ }_{p}$. Since $G_{l}|p=\bar{F}| p, \bar{F}$ can be written as $F \circ\left\langle E_{\text {Exit }}\right\rangle \circ \cdots$, where $F$ is a prefix of $\bar{F}$ such that $p$ executes at least $c+k$ critical events in $F$. However, $p$ and $F$ then satisfy Proposition Pr1, a contradiction. 
Finally, we show that $G_{l}$ satisfies Proposition Pr2. The following derivation establishes (55).

$$
\begin{aligned}
\left|\operatorname{Act}\left(G_{l}\right)\right| & =\left|\operatorname{Inv}_{R F S}\left(G_{l}\right)\right| \\
& \geq\left|\operatorname{Inv}_{R F S}\left(G_{0}\right)\right|-l \\
& =|\operatorname{Act}(\bar{G})-R F S|-l \\
& =\left|Y_{v}-R F S\right|-l \\
& =\left|Y_{v}-(\operatorname{Pmt}(\bar{G}) \cup \operatorname{Fin}(H))\right|-l \\
& =\left|Y_{v}-\operatorname{Pmt}(\bar{G})\right|-l \\
& >\left|Y_{v}\right|-2-2 k \\
& >\sqrt{n}-2 k-3 .
\end{aligned}
$$

\{by $(161), R F S=\operatorname{Fin}\left(G_{l}\right)$, thus $\left.\operatorname{Act}\left(G_{l}\right)=\operatorname{Inv}_{R F S}\left(G_{l}\right)\right\}$

\{by repeatedly applying $(160)\}$

\{by the definition of "Inv"; note that $\left.\bar{G}=G_{0}\right\}$

$\{$ by $(137)\}$

$\{$ because $R F S=\operatorname{Pmt}(\bar{G}) \cup \operatorname{Fin}(\bar{G})$, and

$$
\operatorname{Fin}(\bar{G})=\operatorname{Fin}(H) \text { by }(137)\}
$$

$\left\{\right.$ because $Y_{v} \cap \operatorname{Fin}(H)=\{\}$ by $\left.(137)\right\}$

\{by $(154)$ and $l<2 k\}$

$\{$ by $(112)\}$

Moreover, by (156) and (161), we have $\operatorname{Act}\left(G_{l}\right)=\operatorname{Inv}\left(G_{l}\right)$. Thus, by (137) and (160), we have $\operatorname{Act}\left(G_{l}\right) \subseteq$ $\operatorname{Inv}(\bar{G}) \subseteq \operatorname{Act}(\bar{G})=Y_{v} \subseteq \operatorname{Act}(H)$, which implies (53). By (138) and (161), we have (54). Finally, (158) implies (56). Therefore, $G_{l}$ satisfies Proposition Pr2.

Theorem 2 Let $\bar{N}(k)=(2 k+4)^{2\left(2^{k}-1\right)}$. For any mutual exclusion system $\mathcal{S}=(C, P, V)$ and for any positive number $k$, if $|P| \geq \bar{N}(k)$, then there exists a computation $H$ such that at most $2 k-1$ processes participate in $H$ and some process $p$ executes at least $k$ critical operations in $H$ to enter and exit its critical section.

Proof: Let $H_{1}=\left\langle\right.$ Enter $_{1}$, Enter $_{2}, \ldots$, Enter $\left._{N}\right\rangle$, where $P=\{1,2, \ldots, N\}$ and $N \geq \bar{N}(k)$. By the definition of a mutual exclusion system, $H_{1} \in C$. It is obvious that $H_{1}$ is regular and each process $\operatorname{in} \operatorname{Act}(H)=P$ has exactly one critical event in $H_{1}$. Starting with $H_{1}$, we repeatedly apply Lemma 7 and construct a sequence of computations $\left(H_{1}, H_{2}, \ldots\right)$, such that each process in $\operatorname{Act}\left(H_{j}\right)$ has $j$ critical events in $H_{j}$. We repeat the process until either $H_{k}$ is constructed or some $H_{j}$ satisfies Proposition Pr1 of Lemma 7.

If some $H_{j}(j \leq k-1)$ satisfies Proposition Pr1, then consider the first such $j$. By our choice of $j$, each of $H_{1}, \ldots, H_{j}-1$ satisfies Proposition Pr2 of Lemma 7. Therefore, since $\left|\operatorname{Fin}\left(H_{1}\right)\right|=0$, we have $\mid$ Fin $\left(H_{j}\right) \mid \leq$ $2(j-1) \leq 2 k-4$. It follows that computation $F \circ\left\langle\right.$ Exit $\left._{p}\right\rangle$, generated by applying Lemma 7 to $H_{j}$, satisfies Theorem 2 .

The remaining possibility is that each of $H_{1}, \ldots, H_{k-1}$ satisfies Proposition Pr2. We claim that, for $1 \leq j \leq k$, the following holds:

$$
\left|\operatorname{Act}\left(H_{j}\right)\right| \geq(2 k+4)^{2\left(2^{k+1-j}-1\right)} .
$$

The induction basis $(j=1)$ directly follows from $\operatorname{Act}(H)=P$ and $|P| \geq \bar{N}(k)$. In the induction step, assume that $(162)$ holds for some $j(1 \leq j<k)$, and let $n_{j}=\left|\operatorname{Act}\left(H_{j}\right)\right|$. Note that each active process in $H_{j}$ executes exactly $j$ critical events. By (162), we also have $n_{j}>(2 k+4)^{2}$, which in turn implies that $\sqrt{n_{j}}-2 k-3>\sqrt{n_{j}} /(2 k+4)$. Therefore, by (55), we have

$$
\left|\operatorname{Act}\left(H_{j+1}\right)\right| \geq \min \left(\sqrt{n_{j}} /(2 j+3), \sqrt{n_{j}}-2 k-3\right) \geq \sqrt{n_{j}} /(2 k+4),
$$

from which the induction easily follows.

Finally, (162) implies $\left|\operatorname{Act}\left(H_{k}\right)\right| \geq 1$, and Proposition Pr2 implies $\left|\operatorname{Fin}\left(H_{k}\right)\right| \leq 2(k-1)$. Therefore, select any arbitrary process $p$ from $\operatorname{Act}\left(H_{k}\right)$. Define $G=H_{k} \mid\left(\operatorname{Fin}\left(H_{k}\right) \cup\{p\}\right)$. Clearly, at most $2 k-1$ processes participate in $G$. By applying Lemma 1 with ' $H$ ' $\leftarrow H_{k}$ and ' $Y$ ' $\leftarrow \operatorname{Fin}\left(H_{k}\right) \cup\{p\}$, we have the following: $G \in C$, and an event in $G$ is critical if and only if it is also critical in $H_{k}$. Hence, because $p$ executes $k$ critical events in $H_{k}, G$ is a computation that satisfies Theorem 2. 


\section{Concluding Remarks}

We have established a lower bound that eliminates the possibility of an adaptive mutual exclusion algorithm based on reads, writes, or comparison primitives with $O(\log k)$ RMR time complexity, where $k$ is the highest point (or interval) contention experienced by some active process.

We believe that $\Omega(\min (k, \log N))$ is probably a tight lower bound for the class of algorithms considered in this paper (which would imply that the algorithm in [31] is optimal). One relevant question is whether the results of this paper can be combined with those of [4] or [9] to come close to an $\Omega(\min (k, \log N))$ bound, i.e., can we conclude that $\Omega(\min (k, \log N / \log \log N))$ (for write-update-CC machines) or $\Omega(\min (k, \log N))$ (for DSM and write-invalidate CC machines) is a lower bound? Unfortunately, the answer is no. We have shown that $\Omega(k)$ RMR time complexity is required provided $N$ is sufficiently large. To be specific, for any $k \leq O(\log \log N)$, there exists a computation with contention $O(k)$ in which some process performs $\Omega(k)$ RMRs. This leaves open the possibility that an algorithm might have $\Theta(k)$ RMR time complexity for very "low" levels of contention, but $o(k)$ RMR time complexity for "intermediate" levels of contention. Although our lower bound does not preclude such a possibility, we find it highly unlikely.

It is worth noting that our result pertains to deterministic bounds; one can achieve better expected time complexity with randomized algorithms. For example, Hendler and Woelfel [25] showed that $O(\log k / \log \log k)$ expected amortized RMR time complexity is possible for both DSM and CC machines.

Acknowledgements. We thank Hagit Attiya and the anonymous reviewers for their helpful suggestions throughout the review process.

\section{References}

[1] Y. Afek, H. Attiya, A. Fouren, G. Stupp, and D. Touitou. Long-lived renaming made adaptive. In Proceedings of the 18th Annual ACM Symposium on Principles of Distributed Computing, pages 91-103. ACM, May 1999.

[2] Y. Afek, P. Boxer, and D. Touitou. Bounds on the shared memory requirements for long-lived and adaptive objects. In Proceedings of the 19th Annual ACM Symposium on Principles of Distributed Computing, pages 81-89. ACM, July 2000.

[3] Y. Afek, G. Stupp, and D. Touitou. Long-lived adaptive splitter and applications. Distributed Computing, 15(2):67-86, 2002.

[4] J. Anderson and Y.-J. Kim. An improved lower bound for the time complexity of mutual exclusion. Distributed Computing, 15(4):221-253, December 2003.

[5] J. Anderson, Y.-J. Kim, and T. Herman. Shared-memory mutual exclusion: Major research trends since 1986. Distributed Computing, 16:75-110, 2003.

[6] J. Anderson and J.-H. Yang. Time/contention tradeoffs for multiprocessor synchronization. Information and Computation, 124(1):68-84, January 1996.

[7] H. Attiya and V. Bortnikov. Adaptive and efficient mutual exclusion. Distributed Computing, 15:177-189, 2002. 
[8] H. Attiya, R. Guerraoui, D. Hendler, and P. Kuznetsov. The complexity of obstruction-free implementations. Journal of the ACM, 56:24:1-24:33, July 2009.

[9] H. Attiya, D. Hendler, and P. Woelfel. Tight RMR lower bounds for mutual exclusion and other problems. In Proceedings of the 40th Annual ACM Symposium on Theory of Computing, pages 217-226. ACM, May 2008.

[10] V. Bhatt and C.-C. Huang. Group mutual exclusion in $O(\log n)$ RMR. In Proceeding of the 29th ACM SIGACT-SIGOPS symposium on Principles of distributed computing, PODC '10, pages 45-54. ACM, 2010.

[11] V. Bhatt and P. Jayanti. Constant RMR solutions to reader writer synchronization. In Proceeding of the 29th ACM SIGACT-SIGOPS symposium on Principles of distributed computing, PODC '10, pages 468-477. ACM, 2010.

[12] V. Bhatt and P. Jayanti. Specification and constant RMR algorithm for phase-fair reader-writer lock. In Distributed Computing and Networking, volume 6522 of Lecture Notes in Computer Science, pages 119-130. Springer Berlin / Heidelberg, 2011.

[13] J. Burns and N. Lynch. Mutual exclusion using indivisible reads and writes. In Proceedings of the 18th Annual Allerton Conference on Communication, Control, and Computing, pages 833-842, 1980.

[14] J. Burns and N. Lynch. Bounds on shared memory for mutual exclusion. Information and Computation, 107(2):171-184, December 1993.

[15] M. Choy and A. Singh. Adaptive solutions to the mutual exclusion problem. Distributed Computing, 8(1):1-17, 1994.

[16] R. Cypher. The communication requirements of mutual exclusion. In Proceedings of the Seventh Annual ACM Symposium on Parallel Algorithms and Architectures, pages 147-156. ACM, June 1995.

[17] R. Danek. The $k$-bakery: local-spin $k$-exclusion using non-atomic reads and writes. In Proceeding of the 29th ACM SIGACT-SIGOPS symposium on Principles of distributed computing, PODC '10, pages 36-44. ACM, 2010.

[18] R. Danek and W. Golab. Closing the complexity gap between FCFS mutual exclusion and mutual exclusion. Distributed Computing, 23:87-111, 2010.

[19] R. Danek and V. Hadzilacos. Local-spin group mutual exclusion algorithms. In Proceedings of the 18th International Symposium on Distributed Computing, volume 3274 of Lecture Notes in Computer Science, pages 71-85. Springer Berlin / Heidelberg, 2004.

[20] R. Fan and N. Lynch. An $\Omega(n \log n)$ lower bound on the cost of mutual exclusion. In Proceedings of the twenty-fifth annual ACM symposium on Principles of distributed computing, PODC '06, pages 275-284. ACM, 2006.

[21] W. Golab. A complexity separation between the cache-coherent and distributed shared memory models. In Proceedings of the 30th annual ACM SIGACT-SIGOPS symposium on Principles of distributed computing, PODC '11, pages 109-118. ACM, 2011.

[22] W. Golab, V. Hadzilacos, D. Hendler, and P. Woelfel. Constant-RMR implementations of CAS and other synchronization primitives using read and write operations. In Proceedings of the 26th annual ACM symposium on Principles of distributed computing, PODC '07, pages 3-12. ACM, 2007. 
[23] W. Golab, D. Hendler, and P. Woelfel. An $O(1)$ RMRs leader election algorithm. SIAM Journal on Computing, 39(7):2726-2760, 2010.

[24] D. Hendler and P. Woelfel. Randomized mutual exclusion with sub-logarithmic RMR-complexity. To appear in Distribute Computing; currently available online (a preliminary version appeared in Proceedings of the 28th ACM symposium on Principles of distributed computing, 2009).

[25] D. Hendler and P. Woelfel. Adaptive randomized mutual exclusion in sub-logarithmic expected time. In Proceeding of the 29th ACM SIGACT-SIGOPS symposium on Principles of distributed computing, PODC '10, pages 141-150. ACM, 2010.

[26] M. Herlihy, V. Luchangco, and M. Moir. Obstruction-free synchronization: double-ended queues as an example. In Proceedings of the 23rd IEEE International Conference on Distributed Computing Systems, pages $522-529$, may 2003 .

[27] P. Jayanti. $f$-arrays: implementation and applications. In Proceedings of the twenty-first annual symposium on Principles of distributed computing, PODC '02, pages 270-279. ACM, 2002.

[28] P. Jayanti. Adaptive and efficient abortable mutual exclusion. In Proceedings of the 22nd Annual ACM Symposium on Principles of Distributed Computing, pages 295-304. ACM, 2003.

[29] P. Keane and M. Moir. A simple, local-spin group mutual exclusion algorithm. In Proceedings of the 18th Annual ACM Symposium on Principles of Distributed Computing, pages 23-32. ACM, May 1999.

[30] Y.-J. Kim and J. Anderson. A time complexity bound for adaptive mutual exclusion. In Proceedings of the 15th International Symposium on Distributed Computing, pages 1-15. Lecture Notes in Computer Science 2180, Springer-Verlag, October 2001.

[31] Y.-J. Kim and J. Anderson. Adaptive mutual exclusion with local spinning. Distributed Computing, 19(3):197-236, January 2007.

[32] H. Lee. Transformations of mutual exclusion algorithms from the cache-coherent model to the distributed shared memory model. In Distributed Computing Systems, 2005. ICDCS 2005. Proceedings. 25th IEEE International Conference on, pages $261-270$, june 2005.

[33] J. Mellor-Crummey and M. Scott. Algorithms for scalable synchronization on shared-memory multiprocessors. ACM Transactions on Computer Systems, 9(1):21-65, February 1991.

[34] M. Merritt and G. Taubenfeld. Speeding Lamport's fast mutual exclusion algorithm. Information Processing Letters, 45:137-142, 1993.

[35] E. Styer. Improving fast mutual exclusion. In Proceedings of the 11th Annual ACM Symposium on Principles of Distributed Computing, pages 159-168. ACM, August 1992.

[36] E. Styer and G. Peterson. Tight bounds for shared memory symmetric mutual exclusion. In Proceedings of the 8th Annual ACM Symposium on Principles of Distributed Computing, pages 177-191. ACM, August 1989.

[37] G. Taubenfeld. The black-white bakery algorithm and related bounded-space, adaptive, local-spinning and FIFO algorithms. In Proceedings of the 18th International Symposium on Distributed Computing, volume 3274 of Lecture Notes in Computer Science, pages 56-70. Springer Berlin / Heidelberg, 2004. 
[38] P. Turán. On an extremal problem in graph theory (in Hungarian). Mat. Fiz. Lapok, 48:436-452, 1941.

[39] J.-H. Yang and J. Anderson. A fast, scalable mutual exclusion algorithm. Distributed Computing, 9(1):5160, August 1995. 